\begin{tabular}{|r|l|}
\hline \multicolumn{2}{|c|}{ Statistica Sinica Preprint No: SS-2021-0107 } \\
\hline Title & Statistical Inference for Functional Time Series \\
\hline Manuscript ID & SS-2021-0107 \\
\hline URL & http://www.stat.sinica.edu.tw/statistica/ \\
\hline DOI & $10.5705 /$ ss.202021.0107 \\
\hline Complete List of Authors & Jie Li and \\
& Lijian Yang \\
\hline Corresponding Author & Lijian Yang \\
\hline E-mail & yanglijian@tsinghua.edu.cn \\
\hline Notice: Accepted version subject to English editing.
\end{tabular}


Statistica Sinica

\title{
STATISTICAL INFERENCE FOR FUNCTIONAL TIME SERIES
}

\author{
Jie $\mathrm{Li}^{1}$, Lijian Yang ${ }^{1}$ \\ ${ }^{1}$ Tsinghua University
}

Abstract: Statistical inference is investigated for the mean function of stationary functional time series data with infinite moving average structure. B-spline estimation is proposed for the temporally ordered trajectories of the functional moving average (FMA), which are used to construct a two-step estimator of the mean function. Under mild conditions, the B-spline mean estimator enjoys oracle efficiency in the sense that it is asymptotically equivalent to the infeasible estimator which is the sample mean of all trajectories observed entirely without errors. This oracle efficiency allows for the construction of simultaneous confidence band (SCB) for the mean function which is asymptotically correct. Simulation results strongly corroborate the asymptotic theory. Using the SCB to analyze an ElectroEncephalogram (EEG) time series reveals strong evidence of trigonometric form mean function.

Key words and phrases: B-spline, ElectroEncephalogram, functional moving average, oracle efficiency, simultaneous confidence band. 


\section{Introduction}

Functional data analysis (FDA) has become an area of cutting-edge research for the last two decades, extending statistical analysis of multivariate data to more complicated and informative curve data, see Ferraty and Vieu (2006), Ramsay and Silverman (2002), Ramsay and Silverman (2005),

Hsing and Eubank (2015) and more recently, Kokoszka and Reimherr (2017). Mathematically speaking, classical functional data consists of a collection of $n$ trajectories $\left\{\eta_{t}(\cdot)\right\}_{t=1}^{n}$ corresponding to $n$ subjects, where the $t$-th trajectory $\eta_{t}(\cdot)$ for subject $t$ is a continuous stochastic process equal in distribution to a standard process $\eta(\cdot)$. These trajectories $\left\{\eta_{t}(\cdot)\right\}_{t=1}^{n}$ play the role of univariate and multivariate random observations associated with individual subjects as in most textbooks of introductory statistics. Thus one may be interested in predicting other numerical or categorical outcomes based on such random curves or, at a more basic level, measuring the location and scale of these curves. The latter consists of the mean and covariance functions $m(\cdot)=\mathbb{E}\{\eta(\cdot)\}, G\left(x, x^{\prime}\right)=\operatorname{Cov}\left\{\eta(x), \eta\left(x^{\prime}\right)\right\}$ of $\eta(\cdot)$ respectively, and has been studied in Cao et al. (2012), Cao et al. (2016) and Zheng et al. (2014), where pointwise normal confidence intervals and simultaneous confidence bands (SCBs) have been derived for $m(\cdot)$ and $G(\cdot, \cdot)$ based on various limiting distributions. 
Much of the above is done using "raw" functional data $\left\{Y_{t j}\right\}$ where $Y_{t j}$ represents the discretely recorded value of the $t$-th trajectory $\eta_{t}(\cdot)$ at the $j$-th $x$-location $X_{t j}$, contaminated with measurement error $\sigma\left(X_{t j}\right) \varepsilon_{t j}$,

$$
Y_{t j}=\eta_{t}\left(X_{t j}\right)+\sigma\left(X_{t j}\right) \varepsilon_{t j}, 1 \leq t \leq n, 1 \leq j \leq N_{t}
$$

The "raw data" is therefore not a collection of curves $\left\{\eta_{t}(\cdot)\right\}_{t=1}^{n}$, which may be considered the "smooth data". In the case of densely recorded "raw" data, trajectories $\left\{\eta_{t}(\cdot)\right\}_{t=1}^{n}$ can be estimated one at a time, to produce something that resembles $\left\{\eta_{t}(\cdot)\right\}_{t=1}^{n}$, which might be best referred to as "smoothed pseudo data". To be precise, the "raw data" takes the form

$$
Y_{t j}=\eta_{t}\left(\frac{j}{N}\right)+\sigma\left(\frac{j}{N}\right) \varepsilon_{t j}, 1 \leq t \leq n, 1 \leq j \leq N
$$

where both $N$ and $n$ go to infinity. Spline estimates $\left\{\widehat{\eta}_{t}(\cdot)\right\}_{t=1}^{n}$ are obtained in Cao et al. (2012), the "smoothed pseudo data" that can be used as substitutes for $\left\{\eta_{t}(\cdot)\right\}_{t=1}^{n}$ in data analysis. Without loss of generality, the functions $\eta(\cdot)$ and $\left\{\eta_{t}(\cdot)\right\}_{t=1}^{n}$ are defined on $[0,1], G(\cdot, \cdot)$ on $[0,1]^{2}$. The trajectories $\left\{\eta_{t}(\cdot)\right\}_{t=1}^{n}$ are decomposed as $\eta_{t}(x)=m(x)+\xi_{t}(x)$, where $m(\cdot)$ is continuous on $[0,1], \xi_{t}(x)$ is a small-scale variation of $x$ on the $t$-th trajectory, a process with continuous sample path $\mathbb{E} \xi_{t}(x)=0, \mathbb{E} \max _{x \in[0,1]} \xi_{t}^{2}(x)<\infty$ and continuous covariance $G\left(x, x^{\prime}\right)=\operatorname{Cov}\left\{\xi_{t}(x), \xi_{t}\left(x^{\prime}\right)\right\}$.

According to Hsing and Eubank (2015), there exist eigenvalues $\lambda_{1} \geq$ 
$\lambda_{2} \geq \cdots \geq 0, \sum_{k=1}^{\infty} \lambda_{k}<\infty$, with corresponding eigenfunctions $\left\{\psi_{k}\right\}_{k=1}^{\infty}$ of $G(\cdot, \cdot)$, the latter being an orthonormal basis of $L^{2}[0,1], G\left(x, x^{\prime}\right)=$ $\sum_{k=1}^{\infty} \lambda_{k} \psi_{k}(x) \psi_{k}\left(x^{\prime}\right), \int G\left(x, x^{\prime}\right) \psi_{k}\left(x^{\prime}\right) d x^{\prime}=\lambda_{k} \psi_{k}(x)$. The process $\eta(x)$, $x \in[0,1]$, then allows the well-known Karhunen-Loève $L^{2}$ representation $\eta(x)=m(x)+\sum_{k=1}^{\infty} \xi_{k} \phi_{k}(x)$, in which the random coefficients $\left\{\xi_{k}\right\}_{k=1}^{\infty}$, called functional principal component (FPC) scores, are uncorrelated with mean 0 and variance 1 . The rescaled eigenfunctions $\phi_{k}$ are called FPC, $\phi_{k}=\sqrt{\lambda_{k}} \psi_{k}$, for $k \geq 1$.

Mean estimation is usually the essential first step in functional data analysis; see Ma et al. (2012) and Zheng et al. (2014) for theory and applications of sparse longitudinal data, Cao et al. (2012) for simultaneous confidence band for the mean function based on dense functional data. One serious drawback in Cao et al. (2016) and Cao et al. (2012) is the assumption that the number of records on each curve dominated by the number of curves, namely $N=\mathcal{O}\left(n^{\theta}\right)$ for some $\theta>0$. This constraint is in fact unreasonable, as it prevents observing each subject more densely, whereas by common sense larger $N$ is always more preferable due to increased measurement precision regardless of $n$ being large or small (think of the limiting case $N=\infty$ when observations are made arbitrarily dense in the entire range). In our current work, we have taken instead the assumption 
$n=\mathcal{O}\left(N^{\theta}\right)$, with the more logical and natural understanding that the speed at which $n$ asymptotics kicks in is contingent upon the precision level set by $N$.

These existing works also restricted $\left\{\eta_{t}(\cdot)\right\}_{t=1}^{n}$ to be iid copies of the process $\eta(\cdot)$. As discussed in Bosq (2000), functional data do not always come in the form of iid replicates. One interesting example is the continued recording of EEG (ElectroEncephalogram) for a person in resting eyes-closed state. The participant went through a 5-minute test and EEG signals were recorded at $1000 \mathrm{~Hz}$ sample rate from 32 scalp locations. Observations at the 6 -th location are divided into 400 consecutive segments, each consisting of $N=500$ EEG signals recorded every 0.001 second. Figure 1 shows 5 randomly selected "smoothed pseudo data" from the 400 smooth curves $\left\{\widehat{\eta}_{t}(\cdot)\right\}_{t=1}^{400}$.

Asymptotic theory was developed in Horváth et al. (2013) for testing equality of two mean functions in functional samples exhibiting temporal dependence, on presumption that entire trajectories $\left\{\eta_{t}(\cdot)\right\}_{t=1}^{n}$ are fully observed. Simultaneous confidence bands of the mean function of functional time series was constructed in Chen and Song (2015), but two major gaps existed in its theoretical development: first, it had required the number of positive eigenvalues to be finite, limiting its scope of applicability. Second, it 
had not spelled out clear assumptions on the FPC scores. In particular, its physical dependence condition of (2.1) does not ensure independence of all FPC scores $\xi_{k}, k=1,2, \ldots$, which is a key condition if the Gaussian strong approximations in (A.3) of Lemma A.5 for all $\xi_{t k}, k=1,2, \ldots, k_{n}$ need to be jointly independent so that their linear combinations are Gaussian as well.

To set up an appropriate framework that accounts for dependence among trajectories $\left\{\eta_{t}(\cdot)\right\}_{t=1}^{n}$, the infinite moving average $\mathrm{MA}(\infty)$ concept in classic time series analysis is extended to functional setting. Specifically, the de-meaned trajectories $\left\{\xi_{t}(\cdot)\right\}_{t=1}^{n}$ are regarded as a segment of zero mean processes $\left\{\xi_{t}(\cdot)\right\}_{t=-\infty}^{\infty}$ satisfying the functional moving average $(\operatorname{FMA}(\infty))$ equations

$$
\xi_{t}(\cdot)=\sum_{t^{\prime}=0}^{\infty} A_{t^{\prime}} \zeta_{t-t^{\prime}}(\cdot), t=0, \pm 1, \pm 2, \ldots
$$

in which the $A_{t^{\prime}}$ 's are bounded linear operators $\mathcal{L}^{2}[0,1] \rightarrow \mathcal{L}^{2}[0,1]$ and $\left\{\zeta_{t}(\cdot)\right\}_{t=-\infty}^{\infty}$ are i.i.d. zero mean processes, i.e., strong white noises according to Bosq (2000). Note that classic $\mathrm{MA}(\infty)$ is a rather broad category, which includes as a special case the widely used causal $\operatorname{ARMA}(p, q)$, see Theorem 3.1.1 of Brockwell and Davis (1991).

Under the above dependence structure, B-spline estimator for the mean function $m(\cdot)$ is proposed in the next section. B-spline is widely used in 
nonparametrics for its computational ease as well as conceptional simplicity, see de Boor (2001) and DeVore and Lorentz (1993). It is established in Theorem 2 that the B-spline estimator is as efficient as an infeasible "oracle" estimator which is obtained as if all random trajectories were totally observed without measurement errors. Theorem 1 and Corollary 1 further establish an asymptotically correct SCB of the mean function $m(\cdot)$ under some mild conditions. SCB is a powerful tool of quantifying the variability and making global inferences for functions, see Cao et al. (2016), Cao et al. (2012), Degras (2011) for SCB of dense functional data, Gu et al. (2014), Ma et al. (2012), Zheng et al. (2014) for SCB of sparse functional data, Choi and Reimherr (2017) for ghost region of functional parameters, and Gu and Yang (2015), Wang (2012), Wang and Yang (2009), Zheng et al. (2016), Wang et al. (2020), Yu et al. (2020) for applications and theory of SCB in other contexts. Applying the proposed SCB to the aforementioned EEG data set has revealed strong evidence that the mean function is of simple trigonometric form, see Section 6 .

The paper is organized as follows. Section 2 states main theoretical results on $\mathrm{SCB}$ constructed from B-spline estimator. Section 3 contains a decomposition of the difference between the B-spline estimator and the infeasible one, so that each component can be easily bounded. Procedures to 
implement the proposed SCB are given in Section 4 with details. Section 5 documents simulation findings, and empirical study of the EEG functional time series by the proposed SCB is reported in Section 6. All technical proofs are collected in the Appendix.

\section{Main results}

This section introduces B-spline estimator for the mean function $m(\cdot)$ and studies asymptotic properties of the proposed estimator.

The FMA $(\infty)$ operators $A_{t}$ in (1.3) are of the form

$$
A_{t}\left\{\sum_{k=1}^{\infty} c_{k} \phi_{k}(\cdot)\right\}=\sum_{k=1}^{\infty} a_{t k} c_{k} \phi_{k}(\cdot), a_{t k} \in \mathbb{R}, k=1,2, \ldots, t=0,1, \ldots
$$

with geometrically decaying MA coefficients $\left|a_{t k}\right|<C_{a} \rho_{a}^{t}$ for constants $C_{a}>$ $0, \rho_{a} \in(0,1), k=1,2, \ldots, t=0,1, \ldots$ One notes that geometric decay is not as restrictive as it might seem, since it holds for MA coefficients of causal ARMA model according to equation (3.3.6) of Brockwell and Davis (1991).

The strong functional white noise $\left\{\zeta_{t}(\cdot)\right\}_{t=-\infty}^{\infty}$ allows for its own KarhunenLoève representation $\zeta_{t}(\cdot)=\sum_{k=1}^{\infty} \zeta_{t, k} \phi_{k}(\cdot)$, where $\left\{\zeta_{t, k}\right\}_{t=-\infty, k=1}^{\infty, \infty}$ are uncorrelated random variables with mean 0 and variance 1 . Combining with 
(1.3), one writes

$$
\begin{aligned}
\xi_{t}(\cdot) & =\sum_{t^{\prime}=0}^{\infty} A_{t^{\prime}}\left\{\sum_{k=1}^{\infty} \zeta_{t-t^{\prime}, k} \phi_{k}(\cdot)\right\}=\sum_{t^{\prime}=0}^{\infty} \sum_{k=1}^{\infty} a_{t^{\prime}, k} \zeta_{t-t^{\prime}, k} \phi_{k}(\cdot) \\
& =\sum_{k=1}^{\infty}\left(\sum_{t^{\prime}=0}^{\infty} a_{t^{\prime}, k} \zeta_{t-t^{\prime}, k}\right) \phi_{k}(\cdot),
\end{aligned}
$$

which can be the Karhunen-Loève representation for $\left\{\xi_{t}(\cdot)\right\}_{t=1}^{n}$ as long as each random coefficient $\sum_{t^{\prime}=0}^{\infty} a_{t^{\prime}, k} \zeta_{t-t^{\prime}, k}$ is white noise, i.e., has variance 1. This can be achieved by assuming $\sum_{t=0}^{\infty} a_{t k}^{2} \equiv 1, k=1,2, \ldots$, reasonably analogous to what is assumed in numerical $\mathrm{MA}(\infty)$.

With these in mind and (1.2), the $\operatorname{FMA}(\infty)$ model is

$$
\begin{aligned}
Y_{t j} & =m\left(\frac{j}{N}\right)+\xi_{t}\left(\frac{j}{N}\right)+\sigma\left(\frac{j}{N}\right) \varepsilon_{t j} \\
& =m\left(\frac{j}{N}\right)+\sum_{k=1}^{\infty} \xi_{t k} \phi_{k}\left(\frac{j}{N}\right)+\sigma\left(\frac{j}{N}\right) \varepsilon_{t j}, 1 \leq t \leq n, 1 \leq j \leq(2 N 4)
\end{aligned}
$$

where for $1 \leq t \leq n, k=1,2, \ldots$

$$
\xi_{t}(\cdot)=\sum_{k=1}^{\infty} \xi_{t k} \phi_{k}(\cdot), \xi_{t k}=\sum_{t^{\prime}=0}^{\infty} a_{t^{\prime} k} \zeta_{t-t^{\prime}, k} \quad \text { a.s. }
$$

For any non-negative integer $q$ and fraction $\mu \in(0,1]$, denote by $\mathcal{C}^{(q, \mu)}[0,1]$ the space of functions with $\mu$-Hölder continuous $q$-th derivative, i.e., $\mathcal{C}^{(q, \mu)}[0,1]=\left\{\varphi:[0,1] \rightarrow \mathbb{R}\left|\|\varphi\|_{q, \mu}=\sup _{x, y \in[0,1], x \neq y}\right| \frac{\varphi^{(q)}(x)-\varphi^{(q)}(y)}{|x-y|^{\mu}} \mid<+\infty\right\}$ 
Since $m(\cdot)$ and $\phi_{k}(\cdot)$ both belong to $\mathcal{C}^{(q, \mu)}[0,1]$ under Assumptions (A1) and (A3) below, $\eta(\cdot)$ can be regarded as $\mathcal{C}^{(q, \mu)}[0,1]$-random variables. Had the trajectories $\eta_{t}(\cdot), 1 \leq t \leq n$ been all observed over interval $[0,1]$ entirely, the population mean function $m(\cdot)$ could be estimated by the sample mean of $n$ random variables valued in $\mathcal{C}^{(q, \mu)}[0,1]$

$$
\bar{m}(x)=n^{-1} \sum_{t=1}^{n} \eta_{t}(x), x \in[0,1] .
$$

This "estimator" is infeasible as it makes use of unobservables. It however serves as a benchmark.

To describe the spline functions, one denotes by $\left\{t_{\ell}\right\}_{\ell=1}^{J_{s}}$ a sequence of equally-spaced points, $t_{\ell}=\ell /\left(J_{s}+1\right), 0 \leq \ell \leq J_{s}+1,0=t_{0}<t_{1}<\cdots<$ $t_{J_{s}}<1=t_{J_{s}+1}$, called interior knots, which divide the interval $[0,1]$ into $\left(J_{s}+1\right)$ equal subintervals $I_{\ell}=\left[t_{\ell}, t_{\ell+1}\right), \ell=0, \ldots, J_{s}-1, I_{J_{s}}=\left[t_{J_{s}}, 1\right]$. Let $\mathcal{H}^{(p-2)}=\mathcal{H}^{(p-2)}[0,1]$ be the polynomial spline space of order $p$ on $I_{\ell}, \ell=$ $0, \ldots, J_{s}$, which consists of all $(p-2)$ times continuously differentiable functions on $[0,1]$ that are polynomials of degree $(p-1)$ on subintervals $I_{\ell}, \ell=$ $0, \ldots, J_{s}$. Then, we denote by $\left\{B_{\ell, p}(\cdot), 1 \leq \ell \leq J_{s}+p\right\}$ the $p$ th order B-spline basis functions of $\mathcal{H}^{(p-2)}$, hence $\mathcal{H}^{(p-2)}=\left\{\sum_{\ell=1}^{J_{s}+p} \lambda_{\ell, p} B_{\ell, p}(\cdot) \mid \lambda_{\ell, p} \in \mathbb{R}\right\}$.

The individual trajectories can be estimated via B-spline as

$$
\widehat{\eta}_{t}(\cdot) \equiv \sum_{\ell=1}^{J_{s}+p} \widehat{\beta}_{\ell, p, t} B_{\ell, p}(\cdot), 1 \leq t \leq n
$$


with coefficients $\left\{\widehat{\beta}_{1, p, t}, \ldots, \widehat{\beta}_{J_{s}+p, p, t}\right\}^{\top}$ solving the following least squares problem

$$
\left\{\widehat{\beta}_{1, p, t}, \cdots, \widehat{\beta}_{J_{s}+p, p, t}\right\}^{\top}=\underset{\left\{\beta_{1, p}, \ldots, \beta_{J_{s}+p, p}\right\} \in R^{J_{s}+p}}{\arg \min } \sum_{j=1}^{N}\left\{Y_{t j}-\sum_{\ell=1}^{J_{s}+p} \beta_{\ell, p} B_{\ell, p}(j / N)\right\}^{2} .
$$

The mean function $m(\cdot)$ is then estimated by an oracle estimator

$$
\widehat{m}(\cdot)=n^{-1} \sum_{t=1}^{n} \widehat{\eta}_{t}(\cdot)
$$

which mimicks the infeasible estimator $\bar{m}(\cdot)$ in $(2.6)$.

Throughout the paper, $a_{n} \asymp b_{n}$ means that $a_{n}=\mathcal{O}\left(b_{n}\right)$ and $b_{n}=$ $\mathcal{O}\left(a_{n}\right)$, as $n \rightarrow \infty$. For any measurable function $\phi(\cdot)$ defined on $[0,1]$, denote $\|\phi\|_{\infty}=\sup _{x \in[0,1]}|\phi(x)|$. Denote by $I_{n}$ an integer valued truncation index for the white noise sequence $\zeta_{t k},-\infty<t \leq n$, which satisfies $I_{n}>-10 \log n / \log \rho_{a}, I_{n} \asymp \log n$. We next introduce some technical assumptions.

(A1) The mean function $m(\cdot) \in \mathcal{C}^{(q, \mu)}[0,1]$ for an integer $q>0$ and a constant $\mu \in(0,1]$. One denotes $p^{*}=q+\mu$ in the following.

(A2) The standard deviation function $\sigma(\cdot) \in \mathcal{C}^{(0, \nu)}[0,1]$ for $\nu \in(0,1]$, and $c_{\sigma} \leq \sigma(x) \leq C_{\sigma}, \forall x \in[0,1]$ for constants $0<c_{\sigma}<C_{\sigma}<\infty$

(A3) There exists a constant $\theta>0$, such that as $N \rightarrow \infty, n=n(N) \rightarrow \infty$, $n=\mathcal{O}\left(N^{\theta}\right)$ 
(A4) There exists $C_{G}>0$ such that $G_{\varphi}(x, x) \geq C_{G}, \forall x \in[0,1]$ with $G_{\varphi}(x, x)$ defined in $(2.10)$. The FPCs $\phi_{k}(\cdot) \in \mathcal{C}^{(q, \mu)}[0,1]$ with $\sum_{k=1}^{\infty}\left\|\phi_{k}\right\|_{0, \mu}$ $<+\infty, \sum_{k=1}^{\infty}\left\|\phi_{k}\right\|_{q, \mu}<+\infty, \sum_{k=1}^{\infty}\left\|\phi_{k}\right\|_{\infty}<+\infty$; for increasing positive integers $\left\{k_{n}\right\}_{n=1}^{\infty}$, as $n \rightarrow \infty, \sum_{k_{n}+1}^{\infty}\left\|\phi_{k}\right\|_{\infty}=\mathcal{O}\left(n^{-1 / 2}\right)$ and $k_{n}=\mathcal{O}\left(n^{\omega}\right)$ for some $\omega>0$.

(A5) There are constants $C_{1}, C_{2} \in(0,+\infty), \gamma_{1}, \gamma_{2} \in(1,+\infty), \beta_{1}, \beta_{2} \in$ $(0,1 / 2)$ and iid $N(0,1)$ variables $\left\{Z_{t k, \zeta}\right\}_{t=-I_{n}+1, k=1}^{n, k_{n}},\left\{Z_{t j, \varepsilon}\right\}_{t=1, j=1}^{n, N}$ where $I_{n}$ is the truncation index such that

$$
\begin{array}{r}
\mathbb{P}\left\{\max _{1 \leq k \leq k_{n}-I_{n}+1 \leq \tau \leq n} \max _{t=-I_{n}+1}\left|\zeta_{t k}-\sum_{t=-I_{n}+1}^{\tau} Z_{t k, \zeta}\right|>n^{\beta_{1}}\right\}<C_{1} n^{-\gamma_{1}}, \\
\mathbb{P}\left\{\max _{1 \leq t \leq n} \max _{1 \leq \tau \leq N}\left|\sum_{j=1}^{\tau} \varepsilon_{t j}-\sum_{j=1}^{\tau} Z_{t j, \varepsilon}\right|>N^{\beta_{2}}\right\}<C_{2} N^{-\gamma_{2}} .
\end{array}
$$

(A5') The iid variables $\left\{\varepsilon_{t j}\right\}_{t \geq 1, j \geq 1}$ are independent of FPC score white noises $\left\{\zeta_{t k}\right\}_{t \geq 1, k \geq 1}$. The number of distinct distributions for all FPC score white noises $\left\{\zeta_{t k}\right\}_{t \geq 1, k \geq 1}$ is finite. There exist constants $r_{1}>4+2 \omega$, $r_{2}>4+2 \theta$, for $\omega$ in Assumption (A4) and $\theta$ in Assumption (A3), such that $\mathbb{E}\left|\zeta_{1 k}\right|^{r_{1}}$ and $\mathbb{E}\left|\varepsilon_{11}\right|^{r_{2}}$ are finite.

(A6) The spline order $p \geq p^{*}$, the number of interior knots $J_{s}=N^{\gamma} d_{N}$ for some $\gamma>0$, with $d_{N}+d_{N}^{-1}=\mathcal{O}\left(\log ^{\vartheta} N\right)$ for some $\vartheta>0$ as $N \rightarrow \infty$, and for $p^{*}$ in Assumption (A1), $\nu$ in Assumption (A2), $\theta$ in 
Assumption (A3), $\beta_{2}$ in Assumption (A5) and $r_{1}$ in Assumption (A5')

$$
\max \left\{1-\nu, \frac{\theta}{2 p^{*}}+\frac{2 \theta}{r_{1} p^{*}}\right\}<\gamma<1-\theta / 2-\beta_{2} .
$$

Assumptions (A1) and (A2) are typical for splines smoothing. In particular, (A1) controls the size of the bias of the spline smoother for $m(\cdot)$ and (A2) requires that the variance function is uniformly bounded on its domain. Assumption (A3) restricts that sample size $n$ increases by a fractional power $\theta$ of $N$, the number of observations for each subject. The collective bounded smoothness of the principal components is stated in Assumption (A4). Assumption (A5) provides the Gaussian strong approximation of estimation errors as well as the FPC score white noises $\left\{\zeta_{t k}\right\}_{t=-\infty, k=1}^{\infty, \infty}$. The high level of Assumption (A5) can be ensured by an elementary Assumption (A5'). The requirement for the number of knots of the splines is stipulated in Assumption (A6), which aims to meet for smoothness of B-spline estimator.

Remark 1. The assumptions above are quite mild since they can easily be satisfied in various practical situations. We propose one simple and reasonable setting for the above parameters $q, \mu, \theta, p, \gamma$ as follows: $q+\mu=$ $p^{*}=4, \nu=1, \theta=1, p=4$ (cubic spline), $\gamma=1 / 4, d_{N} \asymp \log \log N$. These constants are used as defaults in implementation, see Section 4. 
Define a limiting covariance function

$$
G_{\varphi}\left(x, x^{\prime}\right)=\sum_{k=1}^{\infty} \phi_{k}(x) \phi_{k}\left(x^{\prime}\right)\left\{1+2 \sum_{t=0}^{\infty} \sum_{t^{\prime}=t+1}^{\infty} a_{t k} a_{t^{\prime} k}\right\}, \quad x, x^{\prime} \in[0,1]
$$

and for i.i.d standard normal variables $\left\{U_{k}\right\}_{k=1}^{\infty}$, denote a Gaussian process

$$
\varphi(x)=\frac{\sum_{k=1}^{\infty} \sum_{t=1}^{\infty} a_{t k} U_{k} \phi_{k}(x)}{G_{\varphi}(x, x)^{1 / 2}}, \quad x \in[0,1] .
$$

Then $\varphi(x)$ satisfies $\mathbb{E} \varphi(x) \equiv 0, \mathbb{E} \varphi^{2}(x) \equiv 1, x \in[0,1]$ with covariance function

$$
\mathbb{E} \varphi(x) \varphi\left(x^{\prime}\right)=G_{\varphi}\left(x, x^{\prime}\right)\left\{G_{\varphi}(x, x) G_{\varphi}\left(x^{\prime}, x^{\prime}\right)\right\}^{-1 / 2}, \quad x, x^{\prime} \in[0,1],
$$

For any $\alpha \in(0,1)$, define $z_{1-\alpha / 2}$ as the $100(1-\alpha / 2)$-th percentile of the standard normal distribution. Denote by $Q_{1-\alpha}$ the $100(1-\alpha)$-th percentile of the absolute maxima distribution of $\varphi(x)$ over $[0,1]$, i.e.,

$$
\mathbb{P}\left[\sup _{x \in[0,1]}|\varphi(x)| \leq Q_{1-\alpha}\right]=1-\alpha
$$

The following result establishes how well $m(\cdot)$ could be estimated had all trajectories $\eta_{t}(\cdot), 1 \leq t \leq n$ been fully observed without error, and used to compute the infeasible "oracle" estimator $\bar{m}(\cdot)$ :

Theorem 1. Under Assumptions (A1), (A3)-(A5), for $\alpha \in(0,1)$, as $n \rightarrow$ $\infty$, the infeasible estimator $\bar{m}(\cdot)$ converges at the $\sqrt{n}$ rate to $m(\cdot)$ with 
asymptotic covariance function $G_{\varphi}\left(x, x^{\prime}\right)$, and thus

$$
\begin{aligned}
\mathbb{P}\left\{\sup _{x \in[0,1]} n^{1 / 2}|\bar{m}(x)-m(x)| G_{\varphi}(x, x)^{-1 / 2} \leq Q_{1-\alpha}\right\} \rightarrow 1-\alpha, & \rightarrow{ }^{2}-\alpha \in[0,1] .
\end{aligned}
$$

The next result enables one to construct simultaneous confidence band based on $\widehat{m}(\cdot)$ in $(2.9)$ by showing that $\widehat{m}(\cdot)$ has the same asymptotic property as $\bar{m}(\cdot)$ in $(2.6)$, so there is no need to differentiate between the two.

Theorem 2. Under Assumptions (A1)-(A6), as $n \rightarrow \infty$, the B-spline estimator $\widehat{m}(\cdot)$ is oracally efficient, i.e., it is asymptotically equivalent to $\bar{m}(\cdot)$ up to order $\mathcal{O}_{p}\left(n^{-1 / 2}\right)$

$$
\sup _{x \in[0,1]} n^{1 / 2}|\bar{m}(x)-\widehat{m}(x)|=\mathcal{O}_{p}(1)
$$

Corollary 1. Under Assumptions (A1)-(A6), for any $\alpha \in(0,1)$, as $n \rightarrow$ $\infty$, an asymptotic $100(1-\alpha) \%$ correct confidence band for $m(\cdot)$ is

$$
\widehat{m}(x) \pm G_{\varphi}(x, x)^{1 / 2} Q_{1-\alpha} n^{-1 / 2}, \quad x \in[0,1]
$$

and an asymptotic $100(1-\alpha) \%$ pointwise confidence interval for $m(x)$ is

$$
\widehat{m}(x) \pm G_{\varphi}(x, x)^{1 / 2} z_{1-\alpha / 2} n^{-1 / 2}, \quad x \in[0,1] .
$$




\section{Decompositon}

In this section, we decompose the estimation error $\widehat{\eta}_{t}(x)-\eta_{t}(x)$ into three convenient terms. For any $L^{2}$ integrable functions $\phi(x)$ and $\varphi(x), x \in$ $[0,1]$, define their theoretical inner product as $\langle\phi, \varphi\rangle=\int_{[0,1]} \phi(x) \varphi(x) d x$, and the empirical inner product as $\langle\phi, \varphi\rangle_{N}=N^{-1} \sum_{j=1}^{N} \phi(j / N) \varphi(j / N)$. The related theoretical and empirical norms are $\|\phi\|_{2}^{2}=\langle\phi, \phi\rangle,\|\phi\|_{2, N}^{2}=$ $\langle\phi, \phi\rangle_{N}$. For any function $\varphi(x)$ defined on $[0,1]$, denote its discretization by $\varphi=\{\varphi(1 / N), \ldots, \varphi(N / N)\}^{\top}$, i.e., the vector of its values on the $N$ measurement points. In particular

$$
\begin{aligned}
& \boldsymbol{\eta}_{t}=\left\{\eta_{t}(1 / N), \ldots, \eta_{t}(N / N)\right\}^{\top}, \mathbf{m}=\{m(1 / N), \ldots, m(N / N)\}^{\top}, \\
& \boldsymbol{\xi}_{t}=\left\{\xi_{t}(1 / N), \ldots, \xi_{t}(N / N)\right\}^{\top}, \boldsymbol{\eta}_{t}=\mathbf{m}+\boldsymbol{\xi}_{t} .
\end{aligned}
$$

Matrix algebra represents the B-spline estimator $\widehat{\eta}_{t}(\cdot)$ in $(2.7)$ as

$$
\widehat{\eta}_{t}(x)=\left\{B_{1, p}(x), \ldots, B_{J_{s}+p, p}(x)\right\}\left(\mathbf{X}^{\top} \mathbf{X}\right)^{-1} \mathbf{X}^{\top} \mathbf{Y}_{t}
$$

where $Y_{t}=\left(Y_{t 1}, \ldots, Y_{t N}\right)^{\top}$ and the design matrix $\mathbf{X}$ is

$$
\mathbf{X}=\left(\begin{array}{ccc}
B_{1, p}(1 / N) & \cdots & B_{J_{s}+p, p}(1 / N) \\
\vdots & \cdots & \vdots \\
B_{1, p}(N / N) & \cdots & B_{J_{s}+p, p}(N / N)
\end{array}\right)_{N \times\left(J_{s}+p\right)} .
$$

Define the empirical inner product matrix of B-spline basis $\left\{B_{\ell, p}(x)\right\}_{\ell=1}^{J_{s}+p}$ 
as

$$
\mathbf{V}_{n, p}=\left\{\left\langle B_{\ell, p}, B_{\ell^{\prime}, p}\right\rangle_{N}\right\}_{\ell, \ell^{\prime}=1}^{J_{s}+p}=N^{-1} \mathbf{X}^{\top} \mathbf{X}
$$

and according to Lemma A.3 in Cao et al. (2012), for some constant $C_{p}>0$,

$$
\left\|\mathbf{V}_{n, p}^{-1}\right\|_{\infty} \leq C_{p} J_{s}
$$

Denote $\boldsymbol{\varepsilon}_{t}=\left\{\sigma(1 / N) \varepsilon_{t 1}, \ldots, \sigma(N / N) \varepsilon_{t N}\right\}^{\top}, \mathbf{B}(x)=\left\{B_{1, p}(x), \ldots, B_{J_{s}+p, p}(x)\right\}^{\top}$, the approximation error $\widehat{\eta}_{t}(x)-\eta_{t}(x)$ is decomposed according to (3.13) as:

$$
\widehat{\eta}_{t}(x)-\eta_{t}(x)=\widetilde{\eta}_{t}(x)-\eta_{t}(x)+\widetilde{\varepsilon}_{t}(x),
$$

where

$$
\begin{aligned}
& \widetilde{\eta}_{t}(x)=N^{-1} \mathbf{B}(x)^{\top} \mathbf{V}_{n, p}^{-1} \mathbf{X}^{\top} \boldsymbol{\eta}_{t}=\widetilde{m}(x)+\widetilde{\xi}_{t}(x) \\
& \widetilde{m}(x)=N^{-1} \mathbf{B}(x)^{\top} \mathbf{V}_{n, p}^{-1} \mathbf{X}^{\top} \mathbf{m}, \quad \widetilde{\xi}_{t}(x)=N^{-1} \mathbf{B}(x)^{\top} \mathbf{V}_{n, p}^{-1} \mathbf{X}^{\top} \boldsymbol{\xi}_{t}, \\
& \widetilde{\varepsilon}_{t}(x)=N^{-1} \mathbf{B}(x)^{\top} \mathbf{V}_{n, p}^{-1} \mathbf{X}^{\top} \boldsymbol{\varepsilon}_{t},
\end{aligned}
$$

Thus, one has $\widehat{\eta}_{t}(x)-\eta_{t}(x)=\widetilde{\xi}_{t}(x)-\xi_{t}(x)+\widetilde{m}(x)-m(x)+\widetilde{\varepsilon}_{t}(x)$. Therefore, by $(2.6)$ and $(2.9)$, the approximation error of $\widehat{m}(\cdot)$ in $(2.9)$ to $\bar{m}(\cdot)$ is

$$
\widehat{m}(x)-\bar{m}(x)=n^{-1} \sum_{t=1}^{n}\left\{\widetilde{\eta}_{t}(x)-\eta_{t}(x)+\widetilde{\varepsilon}_{t}(x)\right\}
$$

\section{Implementation}

This section describes procedures to implement the SCB in Corollary 1. 


\subsection{Knots selection}

\subsection{Knots selection}

The number of knots is an important smoothing parameter, which is selected by AIC.

According to Remark $1, \gamma=1 / 4$ and $d_{N} \asymp \log \log N$ meet Assumption (A6) for $\gamma$ and $d_{N}$, with $J_{s}$ being of order $N^{1 / 4} \log \log N$. Thus we propose to select a data-driven $\widehat{J}_{s}$ from the integers in $\left[0.8 N_{r}, \min \left(10 N_{r}, n / 2\right)\right]$ by AIC, with $N_{r}=N^{1 / 4} \log \log N$. To be more specific, given any data set $\left(j / N, Y_{t j}\right)_{j=1, t=1}^{N, n}$ from model (1.2), denote the estimator for the $j$-th response $Y_{t j}$ by $\widehat{Y}_{t j}\left(N_{n}\right)=\widehat{\eta}_{t}(j / N)$, for $j=1, \ldots, N$. The trajectory estimate $\widehat{\eta}_{t}$ depends on the knot selection sequence as given in (2.7). Then $\widehat{J_{s, t}}$ for $t$-th curve is the one minimizing the $\mathrm{AIC}$ value

$$
\widehat{J_{s, t}}=\underset{N_{n} \in\left[0.8 N_{r}, \min \left(10 N_{r}, n / 2\right)\right]}{\arg \min } \operatorname{AIC}\left(N_{n}\right), \quad t=1, \ldots, n
$$

where $\operatorname{AIC}\left(N_{n}\right)=\log (\mathrm{RSS} / N)+2\left(N_{n}+p\right) / N$, with Residual Sum of Squares RSS $=\sum_{j=1}^{N}\left\{Y_{t j}-\widehat{Y}_{t j}\left(N_{n}\right)\right\}^{2}$. Then $\widehat{J}_{s}$ is set as median of $\left\{\widehat{J_{s, t}}\right\}_{t=1}^{n}$.

The spline estimator $\widehat{\eta}_{t}(\cdot)$ is obtained from (3.14) by using the selected number of knots $\widehat{J}_{s}$, and the estimator $\widehat{m}(\cdot)$ is computed from $(2.9)$. 


\subsection{Covariance estimation}

\subsection{Covariance estimation}

Denote $\widehat{\xi}_{t}(x)=\widehat{\eta}_{t}(x)-\widehat{m}(x), t=1, \ldots, n, x \in[0,1]$. To estimate the covariance function $G_{\varphi}\left(x, x^{\prime}\right)$, one divides $\left\{\widehat{\xi}_{t}(\cdot)\right\}_{t=1}^{n}$ into $l$ groups in order and each group has $B$ samples with $B=\left[n^{1 / 5}\right]$ and $l=[n / B]$, where $[a]$ denotes the integer part of $a$. Noting that $\widehat{G}_{\varphi}(\cdot, \cdot)$ is the limit of the covariance function of the process $\sqrt{n}(\bar{m}(\cdot)-\widehat{m}(\cdot))$, we use $\widehat{m}(x)$ to mimic $m(x)$ and $\sqrt{B} \widehat{\delta}_{j}(x)$ to mimic the points from the process $\sqrt{n}(\bar{m}(\cdot)-\widehat{m}(\cdot))$, where

$$
\widehat{\delta}_{j}(x)=\frac{1}{B} \sum_{k=B(j-1)+1}^{B j} \widehat{\xi}_{k}(x), \quad j=1, \ldots, l, \quad x \in[0,1] .
$$

The estimator $\widehat{G}_{\varphi}\left(x, x^{\prime}\right)$ of $G_{\varphi}\left(x, x^{\prime}\right)$ is defined as

$$
\widehat{G}_{\varphi}\left(x, x^{\prime}\right)=\frac{B}{l} \sum_{j=1}^{l}\left\{\widehat{\delta}_{j}(x) \widehat{\delta}_{j}\left(x^{\prime}\right)-\overline{\widehat{\delta}}(x) \overline{\widehat{\delta}}\left(x^{\prime}\right)\right\}, \quad x, x^{\prime} \in[0,1]
$$

where $\overline{\widehat{\delta}}(x)=l^{-1} \sum_{j=1}^{l} \widehat{\delta}_{j}(x), x \in[0,1]$. Although consistency of $\widehat{G}_{\varphi}\left(x, x^{\prime}\right)$ is straightforward, the proof of which is omitted to save space.

\subsection{Estimating the percentile}

To estimate the percentile $Q_{1-\alpha}$, one first obtains the estimated eigenvalues $\widehat{\lambda}_{k, \varphi}$ and eigenfunctions $\widehat{\psi}_{k, \varphi}$ of $\widehat{G}_{\varphi}\left(x, x^{\prime}\right)$ via $N^{-1} \sum_{j=1}^{N} \widehat{G}_{\varphi}\left(j / N, j^{\prime} / N\right) \widehat{\psi}_{k, \varphi}(j / N)=$ $\widehat{\lambda}_{k, \varphi} \widehat{\psi}_{k, \varphi}\left(j^{\prime} / N\right)$. Next one chooses the number $\kappa$ of eigenfunctions by using 
the following standard criterion, i.e. $\kappa=\underset{1 \leq l \leq T}{\arg \min }\left\{\sum_{k=1}^{l} \widehat{\lambda}_{k, \varphi} / \sum_{k=1}^{T} \widehat{\lambda}_{k, \varphi}>0.95\right\}$, where $\left\{\lambda_{k, \varphi}\right\}_{k=1}^{T}$ are the first $T$ estimated positive eigenvalues.

One then generates $\widehat{\zeta}_{b}(x)=\widehat{G}_{\varphi}(x, x)^{-1 / 2} \sum_{k=1}^{\kappa} Z_{k, b} \widehat{\phi}_{k, \varphi}(x)$, where $\widehat{\phi}_{k, \varphi}=$ $\widehat{\lambda}_{k, \varphi}^{1 / 2} \widehat{\psi}_{k, \varphi}, Z_{k, b}$ are i.i.d standard normal variables with $1 \leq k \leq \kappa$ and $b=1, \ldots, b_{M}$, where $b_{M}$ is a preset large integer with default value 1000 .

One takes the maximal absolute value for each copy of $\widehat{\zeta}_{b}(x)$ and uses the empirical quantile $\widehat{Q}_{1-\alpha}$ of these maximum values as an estimate of $Q_{1-\alpha}$.

Finally, the SCB for the mean function is computed as

$$
\widehat{m}(x) \pm n^{-1 / 2} \widehat{G}_{\varphi}(x, x)^{1 / 2} \widehat{Q}_{1-\alpha}, \quad x \in[0,1]
$$

\section{Simulation}

In this section, simulation studies are conducted to illustrate the finitesample performance of the proposed method. The data is generated from the following model:

$$
Y_{t j}=m(j / N)+\sum_{k=1}^{2} \xi_{t k} \phi_{k}(j / N)+\sigma(j / N) \varepsilon_{t j}, 1 \leq j \leq N, 1 \leq t \leq n .
$$

Case 1: $m(x)=10+\sin \{2 \pi(x-1 / 2)\}, \varepsilon_{t j} \sim N(0,1)$, for $1 \leq t \leq n$, $1 \leq j \leq N, \phi_{1}(x)=-2 \cos \{\pi(x-1 / 2)\}$ and $\phi_{2}(x)=\sin \{\pi(x-1 / 2)\}$. This setting implies $\lambda_{1}=2$ and $\lambda_{2}=0.5 .\left\{\xi_{t k}\right\}_{t=1, k=1}^{n, 2}$ are generated from 
(2.5), where $\left\{\zeta_{t k}\right\}_{t=0, k=1}^{n, 2}$ are i.i.d. $N(0,1)$ variables and

$$
a_{0 k}=0.8, \quad a_{1 k}=0.6, \quad a_{t k}=0, \forall t \geq 2, \quad k=1,2 .
$$

The number of curve $n$ is taken to be 100, 400, 900 and 1600 respectively and the number of observations per curve $N$ is taken to be 120,500 , 1000 and 2000, respectively, while the noise level is set to include both homoscedastic $\sigma(x)=0.3$ and $\sigma(x)=0.5$, as well as heteroscedastic $\sigma(x)=(\exp (x)-0.9) /(\exp (x)+0.9)$ and $\sigma(x)=0.1 \sin (2 \pi x)+0.2$.

Case 2: We set $m(x)=0.4 \sin \{50 \pi(x-1 / 2)\}$ to mimic the data example in Section 6, while $\varepsilon_{t j}, \phi_{1}(x), \phi_{2}(x)$ and $\left\{\xi_{t k}\right\}_{t=1, k=1}^{n, 2}$ are the same as those in Case 1. The number of curve $n$ is taken to be 100, 200, 300 and 400 respectively and the number of observations per curve $N$ is taken to be 500. The noise level is $\sigma=0.005$.

Throughout this section, the mean function is estimated by cubic splines, i.e., $p=4$. Each simulation is repeated 1000 times.

To visualize the SCBs for the mean function, Figure 2 shows the estimated mean functions and their $95 \%$ SCBs for the true curve $m(\cdot)$ in Case 1 when $\sigma=0.3$, and $n=100,400,900,1600$, respectively, with true curve as solid, the estimated curve and the SCBs dashed. As expected, when $n$ increases, the SCB becomes narrower and the cubic spline estimators are closer to the true curve. In all panels, the true mean function is entirely 
covered with SCBs.

Tables 1 to 3 display the empirical coverage rate, namely the percentage out of the 1000 replications of the true curve $m(\cdot)$ being covered by the cubic spline SCBs $(4.25)$ at the $N$ points $\{1 / N, \ldots,(N-1) / N, 1\}$. It is shown in Tables 1 and 2 for Case 1 that regardless of the noise level and/or form, the coverage rate of the SCB becomes closer to the nominal confidence level as the sample size increases. The results in Table 3 for Case 2 are very similar, which reveals a positive confirmation of the asymptotic theory.

\section{Real data analysis}

The SCB methodology is further illustrated with an ElectroEncephalogram (EEG) data collected by research group of Prof. Linhong Ji at Tsinghua University Department of Mechanical Engineering. EEG is known for rich information about brain function. For the study, 145 university students were recruited and EEG signals recorded from 32 scalp locations based on the international 10/20 system of electrode placement. The experiment required participants to go through a 5-minute closed-eye resting state while EEG was being recorded at $1000 \mathrm{~Hz}$ sample rate.

We have selected one person's EEG at the 6-th scalp location, using the mid portion 200000 signals divided into 400 consecutive segments of 
500 recordings each. Each piece can be regraded as a FMA trajectory, with $N=500$ recordings and $n=400$ trajectories. The data range is from -29.2 to 22.8 with an estimated noise level 0.026 , thus the signal to noise ratio is around 2000, close to that of Case 2 in Section 5. While conceding that there are other reasonable choices of $n, N$, we have computed coefficient of determination $R^{2}$ for B spline trajectory against raw EEG data at each of the 400 segments. The sample minimum, 25-th percentile, median, 75th percentile and maximum of the $400 R^{2}$ 's are $0.9993,0.9996,0.9997$, 0.9998 and 0.9999 respectively, showing very good fits for all time $t$. Four randomly selected trajectories are shown in Figure 3, together with the 500 corresponding raw EEG recordings.

The mean function reflects the overall trend of EEG series, and serves as a preliminary step for further data analysis. The mean function of EEG is estimated by (4.25) with cubic spline $(p=4)$ and number of knots chosen by AIC as in Section 4. The accompanying SCB enables testing hypotheses on the mean function, such as certain parametric form. Figure 4 shows that the estimated mean function looks trigonometric, hence the null hypothesis $H_{0}: m(x) \equiv m_{0}(x) \equiv a_{0}+a_{1} \sin (100 \pi x)+b_{1} \cos (100 \pi x)$ is tested, with parameters $a_{0}, a_{1}$ and $b_{1}$ estimated by least square method as $\hat{a}_{0}=-0.0148$, $\hat{a}_{1}=-0.632$ and $\hat{b}_{1}=0.157$. Since the lowest confidence level by which a 
SCB contains the entire the null curve is $2.8 \%$ (see Figure 4), one retains the null hypothesis with a large $p$-value of $1-0.028=0.972$. This suggests strongly that the mean function of the EEG data be of the trigonometric form. We have carried out SCB testing for other participants of the study and reached similar conclusions.

\section{Concluding remarks}

In this paper, a computationally efficient B-spline estimator is proposed for the mean estimation in functional time series. Asymptotic properties of the estimator are established with a simultaneous confidence band (SCB) as a theoretical byproduct, which proves to be a versatile tool of inference on the true mean function. The SCB performs well numerically as well and is illustrated with testing against hypothesis on the possible form of mean function. The FMA $(\infty)$ model can be extended to functional panel data, which promises more interesting discovery of both the mean function and the functional autocovariance function (FACF). The methodology is expected to find wide applications involving physiological data such as EEG and ECG. 


\section{A.1 Preliminaries}

\section{Appendix}

\section{A.1 Preliminaries}

Throughout this section, $\mathcal{O}_{p}$ (or $\mathcal{O}_{p}$ ) denotes a sequence of random variables of certain order in probability. For instance, $\mathcal{O}_{p}\left(n^{-1 / 2}\right)$ means a smaller order than $n^{-1 / 2}$ in probability, and by $\mathcal{O}_{\text {a.s. }}$ (or $\mathcal{O}_{\text {a.s. }}$ ) almost surely $\mathcal{O}$ (or $\mathcal{O})$. In addition, $\mathcal{U}_{p}$ denotes a sequence of random functions which are $\mathcal{O}_{p}$ uniformly defined in the domain.

For any vector $\mathbf{a}=\left(a_{1}, \ldots, a_{n}\right) \in \mathcal{R}^{n}$, denote the norm $\|\mathbf{a}\|_{r}=\left(\left|a_{1}\right|^{r}+\right.$ $\left.\cdots+\left|a_{n}\right|^{r}\right)^{1 / r}, 1 \leq r<+\infty,\|\mathbf{a}\|_{\infty}=\max \left(\left|a_{1}\right|, \ldots,\left|a_{n}\right|\right)$. For any matrix $\mathbf{A}=\left(a_{i j}\right)_{i=1, j=1}^{m, n}$, denote its $L_{r}$ norm as $\|\mathbf{A}\|_{r}=\max _{\mathbf{a} \in \mathcal{R}^{n}, \mathbf{a} \neq \mathbf{0}}\|\mathbf{A} \mathbf{a}\|_{r}\|\mathbf{a}\|_{r}^{-1}$ for $r<+\infty$ and $\|\mathbf{A}\|_{r}=\max _{1 \leq i \leq m} \sum_{j=1}^{n}\left|a_{i j}\right|$, for $r=\infty$. For any random variable $\mathbf{X}$, if it is $L_{p^{-}}$integrable, denotes its $L_{p}$ norm as $\|\mathbf{X}\|_{p}=\left(\mathbb{E}|\mathbf{X}|^{p}\right)^{1 / p}$.

Lemma A.1. (Theorem 2.6.7, Csörgő and Révész (1981)) Suppose that $\xi_{i}, 1 \leq i \leq n$ are iid with $\mathbb{E}\left(\xi_{1}\right)=0, \mathbb{E}\left(\xi_{1}^{2}\right)=1$ and $H(x)>0(x \geq 0)$ is an increasing continuous function such that $x^{-2-\gamma} H(x)$ is increasing for some $\gamma>0$ and $x^{-1} \log H(x)$ is decreasing with $\mathbb{E} H\left(\left|\xi_{1}\right|\right)<\infty$. Then there exist constants $C_{1}, C_{2}, a>0$ which depend only on the distribution of $\xi_{1}$ and a sequence of Brownian motions $\left\{W_{n}(m)\right\}_{n=1}^{\infty}$, such that for any $\left\{x_{n}\right\}_{n=1}^{\infty}$ satisfying $H^{-1}(n)<x_{n}<C_{1}(n \log n)^{1 / 2}$ and $S_{m}=\sum_{i=1}^{m} \xi_{i}$, then 


\section{A.1 Preliminaries}

$\mathbb{P}\left\{\max _{1 \leq m \leq n}\left|S_{m}-W_{n}(m)\right|>x_{n}\right\} \leq C_{2} n\left\{H\left(a x_{n}\right)\right\}^{-1}$.

Lemma A.2. (Theorem 7.5, Billingsley (1999)) Let $(\Omega, \mathcal{F}, \mathbb{P})$ be a probability space and let $X$ map $\Omega$ into $C[0,1]: X(\omega)$ is an element of $C[0,1]$ with value $X_{t}(\omega)=X(t, \omega)$ at $t$. For $F \in C[0,1]$, denote $\omega(F, h)=$ $\sup _{x, x^{\prime} \in[0,1],\left|x-x^{\prime}\right| \leq h}$ $\left|F\left(x^{\prime}\right)-F(x)\right|$ as the modulus of continuity. Suppose that $X, X^{1}, X^{2}, \ldots$ are random functions. If $\left(X_{t_{1}}^{n}, \ldots, X_{t_{k}}^{n}\right) \rightarrow_{D}\left(X_{t_{1}}, \ldots, X_{t_{k}}\right)$ holds for all $t_{1}, \ldots, t_{k}$, and if

$$
\lim _{\delta \rightarrow 0} \limsup _{n \rightarrow \infty} \mathbb{P}\left[\omega\left(X^{n}, \delta\right) \geq \epsilon\right]=0
$$

for each positive $\epsilon$, then $X^{n} \rightarrow_{D} X$.

Lemma A.3. For $n>2, a>2, W_{i} \sim N\left(0, \sigma_{i}^{2}\right), \sigma_{i}>0, i=1, \ldots, n$

$$
\mathbb{P}\left(\max _{1 \leq i \leq n}\left|W_{i} / \sigma_{i}\right|>a \sqrt{\log n}\right)<\sqrt{\frac{2}{\pi}} n^{1-a^{2} / 2} .
$$

As $n \rightarrow \infty,\left(\max _{1 \leq i \leq n}\left|W_{i}\right|\right) /\left(\max _{1 \leq i \leq n} \sigma_{i}\right) \leq \max _{1 \leq i \leq n}\left|W_{i} / \sigma_{i}\right|=\mathcal{O}_{a . s .}(\sqrt{\log n})$.

Proof. Note that

$$
\begin{aligned}
\mathbb{P}\left(\max _{1 \leq i \leq n}\left|\frac{W_{i}}{\sigma_{i}}\right|>a \sqrt{\log n}\right) & \leq \sum_{i=1}^{n} \mathbb{P}\left(\left|\frac{W_{i}}{\sigma_{i}}\right|>a \sqrt{\log n}\right) \leq 2 n\{1-\Phi(a \sqrt{\log n})\} \\
& <2 n \frac{\phi(a \sqrt{\log n})}{a \sqrt{\log n}} \leq 2 n \phi(a \sqrt{\log n})=\sqrt{\frac{2}{\pi}} n^{1-a^{2} / 2}
\end{aligned}
$$

for $n>2, a>2$, which proves (A.2). The lemma follows by applying Borel-Cantelli Lemma with choice of $a>2$. 


\section{A.1 Preliminaries}

Lemma A.4. Assumption (A5) holds under Assumptions (A3), (A4) and $\left(A 5^{\prime}\right)$.

Proof. Under Assumption (A5'), $\mathbb{E}\left|\zeta_{t k}\right|^{r_{1}}<\infty, r_{1}>4+2 \omega$, $\mathbb{E}\left|\varepsilon_{t j}\right|^{r_{2}}<\infty, r_{2}>4+2 \theta$, where $\omega$ is defined in Assumption (A4) and $\theta$ is defined in Assumption (A3), so there exists some $\beta_{0}, \beta_{1}, \beta_{2} \in(0,1 / 2)$, such that $r_{1}>(2+\omega) / \beta_{0}, r_{2}>(2+\theta) / \beta_{2}$.

Let $H(x)=x^{r_{1}}$. Lemma A.1 entails that there exist constants $c_{1 k}$ and $a_{k}$ depending on the distribution of $\zeta_{t k}$, such that for $x_{n}=\left(n+I_{n}\right)^{\beta_{0}}$, $\left(n+I_{n}\right) / H\left(a_{k} x_{n}\right)=a_{k}^{-r_{1}}\left(n+I_{n}\right)^{1-r_{1} \beta_{0}}$ and iid $N(0,1)$ variables $Z_{t k, \zeta}$, $\mathbb{P}\left\{\max _{-I_{n}+1 \leq \tau \leq n}\left|\sum_{t=-I_{n}+1}^{\tau} \zeta_{t k}-\sum_{t=-I_{n}+1}^{\tau} Z_{t k, \zeta}\right|>\left(n+I_{n}\right)^{\beta_{0}}\right\}<c_{1 k} a_{k}^{-r_{1}}\left(n+I_{n}\right)^{1-r_{1} \beta_{0}}$,

Since there are only a finite number of distinct distributions for $\left\{\zeta_{t k}\right\}_{t=-I_{n}+1, k=1}^{n, k_{n}}$ by Assumption (A5'), there exists a common $c_{1}>0$, such that $\max _{1 \leq k \leq k_{n}} \mathbb{P}\left\{\max _{-I_{n}+1 \leq \tau \leq n}\left|\sum_{t=-I_{n}+1}^{\tau} \zeta_{t, k}-\sum_{t=-I_{n}+1}^{\tau} Z_{t k, \zeta}\right|>\left(n+I_{n}\right)^{\beta_{0}}\right\}<c_{1}\left(n+I_{n}\right)^{1-r_{1} \beta_{0}}$.

Since $I_{n} \asymp \log n$ by definition, there exists $\epsilon>\beta_{0} \log \left\{\left(n+I_{n}\right) / n\right\} / \log n$, such that $n^{\beta_{0}+\epsilon}>\left(n+I_{n}\right)^{\beta_{0}}$. Noting that $\beta_{0} \log \left\{\left(n+I_{n}\right) / n\right\} / \log n \rightarrow 0$ as $n \rightarrow \infty$, one can choose $\epsilon<1 / 2-\beta_{0}$. Denote $\beta_{1}=\beta_{0}+\epsilon$, then $\beta_{1}<1 / 2$ and $n^{\beta_{1}}>\left(n+I_{n}\right)^{\beta_{0}}$. Because $1-r_{1} \beta_{0}<0$, it is clear that $\left(n+I_{n}\right)^{1-r_{1} \beta_{0}}<n^{1-r_{1} \beta_{0}}$. Thus one has

$$
\max _{1 \leq k \leq k_{n}} \mathbb{P}\left\{\max _{-I_{n}+1 \leq \tau \leq n}\left|\sum_{t=-I_{n}+1}^{\tau} \zeta_{t, k}-\sum_{t=-I_{n}+1}^{\tau} Z_{t k, \zeta}\right|>n^{\beta_{1}}\right\}<c_{1} n^{1-r_{1} \beta_{0}} .
$$




\section{A.1 Preliminaries}

Recalling that $r_{1}>(2+\omega) / \beta_{0}$, one can let $\gamma_{1}=r_{1} \beta_{0}-1-\omega>1$, and there exists a $C_{1}>0$ such that

$\mathbb{P}\left\{\max _{1 \leq k \leq k_{n}} \max _{-I_{n}+1 \leq \tau \leq n}\left|\sum_{t=-I_{n}+1}^{\tau} \zeta_{t, k}-\sum_{t=-I_{n}+1}^{\tau} Z_{t k, \zeta}\right|>n^{\beta_{1}}\right\}<k_{n} c_{1} n^{1-r_{1} \beta_{0}} \leq C_{1} n^{-\gamma_{1}}$.

Likewise, under Assumption (A5'), taking $H(x)=x^{r_{2}}$, Lemma A.1 implies that there exists constants $c_{2}$ and $b$ depending on the distribution of $\varepsilon_{i j}$, such that for $x_{N}=N^{\beta_{2}}, N / H\left(b x_{N}\right)=b^{-r_{2}} N^{1-r_{2} \beta_{2}}$ and iid standard normal random variables $\left\{Z_{t j, \varepsilon}\right\}_{t=1, j=1}^{n, N}$ such that

$$
\max _{1 \leq t \leq n} \mathbb{P}\left\{\max _{1 \leq \tau \leq N}\left|\sum_{j=1}^{\tau} \varepsilon_{t j}-\sum_{j=1}^{\tau} Z_{t j, \varepsilon}\right|>N^{\beta_{2}}\right\}<c_{2} b^{-r_{2}} N^{1-r_{2} \beta_{2}}
$$

Assumption (A3) states that $n=\mathcal{O}\left(N^{\theta}\right)$, so there is a $C_{2}>0$ such that

$\mathbb{P}\left\{\max _{1 \leq t \leq n} \max _{1 \leq \tau \leq N}\left|\sum_{j=1}^{\tau} \varepsilon_{t j}-\sum_{j=1}^{\tau} Z_{t j, \varepsilon}\right|>N^{\beta_{2}}\right\}<c_{2} b^{-r_{2}} n \times N^{1-r_{2} \beta_{2}} \leq C_{2} N^{\theta+1-r_{2} \beta_{2}}$.

Since $r_{2} \beta_{2}>(2+\theta)$, there is $\gamma_{2}=r_{2} \beta_{2}-1-\theta>1$ and Assumption (A5)

follows.

Lemma A.5. Under Assumptions (A5) and (A5'), as $n \rightarrow \infty$, there exist $C_{3}, C_{4} \in(0,+\infty), \gamma_{3} \in(1,+\infty), \beta_{3} \in(0,1 / 2)$ and $N(0,1)$ variables $Z_{t k, \xi}=$ $\sum_{t^{\prime}=0}^{\infty} a_{t^{\prime} k} Z_{t-t^{\prime}, k, \zeta}, t=1, \ldots, n, k=1, \ldots, k_{n}$, with $Z_{t k, \zeta}$ 's as in Lemma

A.4. Consequently for $1 \leq j \leq n, 1 \leq h \leq n-j \operatorname{Cov}\left(Z_{j k, \xi}, Z_{j+h, k, \xi}\right)=$ $\sum_{m=0}^{\infty} a_{m k} a_{m+h, k}$ and one has

$$
\mathbb{P}\left\{\max _{1 \leq k \leq k_{n}} \max _{1 \leq \tau \leq n}\left|\sum_{t=1}^{\tau} \xi_{t k}-\sum_{t=1}^{\tau} Z_{t k, \xi}\right|>C_{3} n^{\beta_{3}}\right\}<C_{4} n^{-\gamma_{3}} .
$$




\section{A.1 Preliminaries}

Proof. Since $\sum_{t=0}^{\infty} a_{t k}^{2}=1$ and $\left|a_{t k}\right|<C_{a} \rho_{a}^{t}$, for $t=0, \ldots, n$, $k=1, \ldots, k_{n}$, together with $I_{n}>-10 \log n / \log \rho_{a}$ in Assumption (A5), then $\rho_{a}^{I_{n}}<n^{-10}, \rho_{a}^{t^{\prime}-I_{n}}$, when $t^{\prime}>I_{n}$ and $\sum_{t=0}^{I_{n}}\left|a_{t k}\right|<M$ for some constant $M>0$. It is clear that

$$
\begin{array}{r}
\xi_{t k}=\sum_{t^{\prime}=0}^{I_{n}} a_{t^{\prime} k} \zeta_{t-t^{\prime}, k}+\sum_{t^{\prime}=I_{n}+1}^{\infty} a_{t^{\prime} k} \zeta_{t-t^{\prime}, k}, \\
\left|\xi_{t k}-\sum_{t^{\prime}=0}^{I_{n}} a_{t^{\prime} k} \zeta_{t-t^{\prime}, k}\right| \leq \sum_{t^{\prime}=I_{n}+1}^{\infty} C_{a} n^{-10} \rho_{a}^{t^{\prime}-I_{n}}\left|\zeta_{t-t^{\prime}, k}\right|, \\
\left|\xi_{t k}-\sum_{t^{\prime}=0}^{I_{n}} a_{t^{\prime} k} \zeta_{t-t^{\prime}, k}\right| \leq C_{a} n^{-10} \sum_{t^{\prime}=1}^{\infty} \rho_{a}^{t^{\prime}}\left|\zeta_{t-I_{n}-t^{\prime}, k}\right| .
\end{array}
$$

Hence,

$\max _{1 \leq k \leq k_{n}} \max _{1 \leq \tau \leq n}\left|\sum_{t=1}^{\tau} \xi_{t k}-\sum_{t=1}^{\tau} \sum_{t^{\prime}=0}^{I_{n}} a_{t^{\prime} k} \zeta_{t-t^{\prime}, k}\right| \leq \max _{1 \leq k \leq k_{n}} \max _{1 \leq t \leq n} C_{a} n^{-9} \sum_{t^{\prime}=1}^{\infty} \rho_{a}^{t^{\prime}}\left|\zeta_{t-I_{n}-t^{\prime}, k}\right|$

Denote $W_{t k}=\sum_{t^{\prime}=1}^{\infty} \rho_{a}^{t^{\prime}}\left|\zeta_{t-I_{n}-t^{\prime}, k}\right|$ and note that $\sup _{t, k} \mathbb{E}\left|\zeta_{t, k}\right|^{r_{1}}<\infty$, where $r_{1}>4+2 \omega$,

$$
\left\|W_{t k}\right\|_{r_{1}} \leq \sum_{t^{\prime}=1}^{\infty} \rho_{a}^{t^{\prime}}\left\|\zeta_{t-I_{n}-t^{\prime}, k}\right\|_{r_{1}}<\infty
$$

Therefore, $\mathbb{E} W_{t k}^{r_{1}}<K$ for some $K>0, t=1, \ldots, n, k=1, \ldots, k_{n}$. Note that $k_{n}=\mathcal{O}\left(n^{\omega}\right)$ in Assumption (A4), thus

$\mathbb{P}\left(C_{a} n^{-9} \max _{1 \leq k \leq k_{n}} \max _{1 \leq t \leq n} W_{t k}>M n^{\beta_{3}}\right)<n k_{n} \frac{C_{a}^{r_{1}} K}{M^{r_{1}}} n^{-\left(\beta_{3}+9\right) r_{1}}<\frac{C_{a}^{r_{1}} K}{M^{r_{1}}} n^{-\left(\beta_{3}+9\right) r_{1}+1+\omega}$.

So,

$\mathbb{P}\left[\max _{1 \leq k \leq k_{n}} \max _{1 \leq \tau \leq n}\left|\sum_{t=1}^{\tau} \xi_{t k}-\sum_{t=1}^{\tau} \sum_{t^{\prime}=0}^{I_{n}} a_{t^{\prime} k} \zeta_{t-t^{\prime}, k}\right|>M n^{\beta_{3}}\right]<\frac{C_{a}^{r_{1}} K}{M^{r_{1}}} n^{-\left(\beta_{3}+9\right) r_{1}+1+\omega}$. 


\section{A.1 Preliminaries}

Next, define $U_{t k}=\sum_{t^{\prime}=I_{n}+1}^{\infty} a_{t^{\prime} k} Z_{t-t^{\prime}, k, \zeta}$, then $U_{t k} \sim N\left(0, \sum_{t^{\prime}=I_{n}+1}^{\infty} a_{t^{\prime} k}^{2}\right)$, $k=1, \ldots, k_{n}$. It is obvious that

$$
\max _{1 \leq k \leq k_{n}} \max _{1 \leq \tau \leq n}\left|\sum_{t=1}^{\tau} \sum_{t^{\prime}=I_{n}+1}^{\infty} a_{t^{\prime} k} Z_{t-t^{\prime}, k, \zeta}\right| \leq n \max _{1 \leq k \leq k_{n}} \max _{1 \leq t \leq n}\left|U_{t k}\right| .
$$

Note that $\sum_{t^{\prime}=I_{n}+1}^{\infty} a_{t^{\prime} k}^{2}<C n^{-20}$ for some $C>0, k=1, \ldots, k_{n}$ and $k_{n}=$ $\mathcal{O}\left(n^{\omega}\right)$ for some $\omega>0$, one has

$$
\mathbb{P}\left(n \max _{1 \leq k \leq k_{n}} \max _{1 \leq t \leq n}\left|U_{t k}\right|>M n^{\beta_{3}}\right)<n k_{n} \frac{C n^{-20}}{M^{2}\left(n^{\beta_{3}-1}\right)^{2}}<\frac{C}{M^{2}} n^{-17-2 \beta_{3}+\omega}
$$

which leads to

$\mathbb{P}\left[\max _{1 \leq k \leq k_{n}} \max _{1 \leq \tau \leq n}\left|\sum_{t=1}^{\tau} \sum_{t^{\prime}=I_{n}+1}^{\infty} a_{t^{\prime} k} Z_{t-t^{\prime}, k, \zeta}\right|>M n^{\beta_{3}}\right]<n k_{n} \frac{C n^{-20}}{M^{2}\left(n^{\beta_{3}-1}\right)^{2}}<\frac{C}{M^{2}} n^{-17-2 \beta_{3}+\omega}$.

Now Assumption (A5) entails that for $0 \leq t^{\prime} \leq I_{n}, 1 \leq t \leq n,-I_{n}+1 \leq$

$t-t^{\prime} \leq n$

$$
\mathbb{P}\left\{\max _{1 \leq k \leq k_{n}-I_{n}+1 \leq \tau \leq n}\left|\sum_{t=-I_{n}+1}^{\tau} \zeta_{t k}-\sum_{t=-I_{n}+1}^{\tau} Z_{t k, \zeta}\right|>n^{\beta_{3}}\right\}<C_{1} n^{-\gamma_{1}} .
$$

Then,

$$
\begin{aligned}
& \mathbb{P}\left[\max _{1 \leq k \leq k_{n}} \max _{1 \leq \tau \leq n}\left|\sum_{t=1}^{\tau} \sum_{t^{\prime}=0}^{I_{n}} a_{t^{\prime} k}\left(\zeta_{t-t^{\prime}, k}-Z_{t-t^{\prime}, k, \zeta}\right)\right|>2 M n^{\beta_{3}}\right] \\
= & \mathbb{P}\left[\max _{1 \leq k \leq k_{n}} \max _{1 \leq \tau \leq n}\left|\sum_{t^{\prime}=0}^{I_{n}} a_{t^{\prime} k} \sum_{t=1}^{\tau}\left(\zeta_{t-t^{\prime}, k}-Z_{t-t^{\prime}, k, \zeta}\right)\right|>2 M n^{\beta_{3}}\right] \\
\leq & \mathbb{P}\left[\max _{1 \leq k \leq k_{n}}\left\{\sum_{t^{\prime}=0}^{I_{n}}\left|a_{t^{\prime} k}\right| \max _{1 \leq \tau \leq n}\left|\sum_{t=1}^{\tau} \zeta_{t-t^{\prime}, k}-\sum_{t=1}^{\tau} Z_{t-t^{\prime}, k, \zeta}\right|\right\}>2 M n^{\beta_{3}}\right]
\end{aligned}
$$




\section{A.1 Preliminaries}

$$
\begin{aligned}
& \leq \mathbb{P}\left[M \max _{1 \leq k \leq k_{n}} \max _{1 \leq \tau \leq n} \max _{0 \leq t^{\prime} \leq I_{n}}\left|\sum_{t=1}^{\tau} \zeta_{t-t^{\prime}, k}-\sum_{t=1}^{\tau} Z_{t-t^{\prime}, k, \zeta}\right|>2 M n^{\beta_{3}}\right] \\
& \leq \mathbb{P}\left\{2 \max _{1 \leq k \leq k_{n}} \max _{1 \leq \tau \leq n}\left|\sum_{t=-I_{n}+1}^{\tau} \zeta_{t k}-\sum_{t=-I_{n}+1}^{\tau} Z_{t k, \zeta}\right|>2 n^{\beta_{3}}\right\}<C_{1} n^{-\gamma_{1}}
\end{aligned}
$$

Hence,

$$
\begin{aligned}
& \mathbb{P}\left[\max _{1 \leq k \leq k_{n}} \max _{1 \leq \tau \leq n}\left|\sum_{t=1}^{\tau} \xi_{t k}-\sum_{t=1}^{\tau}\left(\sum_{t^{\prime}=0}^{\infty} a_{t^{\prime} k} Z_{t-t^{\prime}, k, \zeta}\right)\right|>4 M n^{\beta_{3}}\right] \\
& =\mathbb{P}\left[\max _{1 \leq k \leq k_{n}} \max _{1 \leq \tau \leq n} \mid \sum_{t=1}^{\tau} \xi_{t k}-\sum_{t=1}^{\tau} \sum_{t^{\prime}=0}^{I_{n}} a_{t^{\prime} k} \zeta_{t-t^{\prime}, k}+\sum_{t=1}^{\tau} \sum_{t^{\prime}=0}^{I_{n}} a_{t^{\prime} k} \zeta_{t-t^{\prime}, k}-\sum_{t=1}^{\tau} \sum_{t^{\prime}=0}^{I_{n}} a_{t^{\prime} k} Z_{t-t^{\prime}, k, \zeta}\right. \\
& \left.-\sum_{t=1}^{\tau} \sum_{t^{\prime}=I_{n}+1}^{\infty} a_{t^{\prime} k} Z_{t-t^{\prime}, k, \zeta} \mid>4 M n^{\beta_{3}}\right] \\
& \leq \mathbb{P}\left[\operatorname { m a x } _ { 1 \leq k \leq k _ { n } } \operatorname { m a x } _ { 1 \leq \tau \leq n } \left\{\left|\sum_{t=1}^{\tau} \xi_{t k}-\sum_{t=1}^{\tau} \sum_{t^{\prime}=0}^{I_{n}} a_{t^{\prime} k} \zeta_{t-t^{\prime}, k}\right|+\left|\sum_{t=1}^{\tau} \sum_{t^{\prime}=0}^{I_{n}} a_{t^{\prime} k} \zeta_{t-t^{\prime}, k}-\sum_{t=1}^{\tau} \sum_{t^{\prime}=0}^{I_{n}} a_{t^{\prime} k} Z_{t-t^{\prime}, k, \zeta}\right|\right.\right. \\
& \left.\left.+\left|\sum_{t=1}^{\tau} \sum_{t^{\prime}=I_{n}+1}^{\infty} a_{t^{\prime} k} Z_{t-t^{\prime}, k, \zeta}\right|\right\}>4 M n^{\beta_{3}}\right] \\
& \leq \mathbb{P}\left[\max _{1 \leq k \leq k_{n}} \max _{1 \leq \tau \leq n}\left|\sum_{t=1}^{\tau} \xi_{t k}-\sum_{t=1}^{\tau} \sum_{t^{\prime}=0}^{I_{n}} a_{t^{\prime} k} \zeta_{t-t^{\prime}, k}\right|>M n^{\beta_{3}}\right]+\mathbb{P}\left[\max _{1 \leq k \leq k_{n}} \max _{1 \leq \tau \leq n} \mid \sum_{t=1}^{\tau} \sum_{t^{\prime}=0}^{I_{n}} a_{t^{\prime} k} \zeta_{t-t^{\prime}, k}\right. \\
& \left.-\sum_{t=1}^{\tau} \sum_{t^{\prime}=0}^{I_{n}} a_{t^{\prime} k} Z_{t-t^{\prime}, k, \zeta} \mid>2 M n^{\beta_{3}}\right]+\mathbb{P}\left[\max _{1 \leq k \leq k_{n}} \max _{1 \leq \tau \leq n}\left|\sum_{t=1}^{\tau} \sum_{t^{\prime}=I_{n}+1}^{\infty} a_{t^{\prime} k} Z_{t-t^{\prime}, k, \zeta}\right|>M n^{\beta_{3}}\right] \\
& \leq \frac{C_{a}^{r_{1}} K}{M^{r_{1}}} n^{-\left(\beta_{3}+9\right) r_{1}+1+\omega}+\frac{C}{M^{2}} n^{-17-2 \beta_{3}+\omega}+C_{1} n^{-\gamma_{1}}<C_{4} n^{-\gamma_{3}}
\end{aligned}
$$

Denote $C_{3}=4 M$ and $Z_{t k, \xi}=\sum_{t^{\prime}=0}^{\infty} a_{t^{\prime} k} Z_{t-t^{\prime}, k, \zeta}, t=1, \ldots, n, k=1, \ldots, k_{n}$,

then $\left\{Z_{t k, \xi}\right\}_{t=1, k=1}^{n, k_{n}}$ are $N(0,1)$ variables and $\operatorname{Cov}\left(Z_{j, k, \xi}, Z_{j+h, k, \xi}\right)=\sum_{m=0}^{\infty} a_{m k} a_{m+h, k}, 1 \leq$ $j \leq n, 1 \leq h \leq n-j$, thus

$$
\mathbb{P}\left[\max _{1 \leq k \leq k_{n}} \max _{1 \leq \tau \leq n}\left|\sum_{t=1}^{\tau} \xi_{t k}-\sum_{t=1}^{\tau} Z_{t k, \xi}\right|>C_{3} n^{\beta_{3}}\right]<C_{4} n^{-\gamma_{3}} .
$$




\section{A.1 Preliminaries}

The proof is completed.

Lemma A.6. Under Assumptions (A2), (A5) and (A6), as $n \rightarrow \infty$

$$
\max _{1 \leq \ell \leq J_{s}+p}\left|(n N)^{-1} \sum_{t=1}^{n} \sum_{j=1}^{N} B_{\ell, p}(j / N) \sigma(j / N) Z_{t j, \varepsilon}\right|=\mathcal{O}_{a . s .}\left(n^{-1 / 2} N^{-1 / 2} J_{s}^{-1 / 2} \log ^{1 / 2} N\right) .
$$

Proof. Note that $(n N)^{-1} \sum_{t=1}^{n} \sum_{j=1}^{N} B_{\ell, p}(j / N) \sigma(j / N) Z_{t j, \varepsilon}=N^{-1} \sum_{j=1}^{N} B_{\ell, p}(j / N)$ $\sigma(j / N) Z_{\cdot j, \varepsilon}$, where $Z_{\cdot j, \varepsilon}=n^{-1} \sum_{t=1}^{n} Z_{t j, \varepsilon}$, one can apply Lemma A.3 to obtain uniform bound for zero mean Gaussian variables $N^{-1} \sum_{j=1}^{N} B_{\ell, p}(j / N) \sigma(j / N) Z_{\cdot j, \varepsilon}$, $1 \leq \ell \leq J_{s}+p$ with variance

$$
\begin{aligned}
\mathbb{E}\left\{N^{-1} \sum_{j=1}^{N} B_{\ell, p}(j / N) \sigma(j / N) Z_{\cdot j, \varepsilon}\right\}^{2} & =n^{-1} N^{-2} \sum_{j=1}^{N} B_{\ell, p}^{2}\left(\frac{j}{N}\right) \sigma^{2}\left(\frac{j}{N}\right) \\
& =n^{-1} N^{-1}\left\|B_{\ell, p} \sigma\right\|_{2, N}^{2} \asymp J_{s}^{-1} N^{-1} n^{-1}
\end{aligned}
$$

It follows from Lemma A.3 that

$$
\begin{aligned}
\max _{1 \leq \ell \leq J_{s}+p}\left|N^{-1} \sum_{j=1}^{N} B_{\ell, p}(j / N) \sigma(j / N) Z_{\cdot j, \varepsilon}\right| & =\mathcal{O}_{a . s .}\left\{n^{-1 / 2} N^{-1 / 2} J_{s}^{-1 / 2} \log ^{1 / 2}\left(J_{s}+p\right)\right\} \\
& =\mathcal{O}_{\text {a.s. }}\left(n^{-1 / 2} N^{-1 / 2} J_{s}^{-1 / 2} \log ^{1 / 2} N\right)
\end{aligned}
$$

where the last step follows from Assumption (A6) on the order of $J_{s}$ relative to $N$. Thus the lemma holds.

Lemma A.7. Under Assumptions (A2), (A5) and (A6), as $n \rightarrow \infty$

$$
\sup _{x \in[0,1]} n^{-1}\left|\sum_{t=1}^{n} \widetilde{\varepsilon}_{t}(x)\right|=\mathcal{O}_{a . s .}\left(n^{-1 / 2} N^{-1 / 2} J_{s}^{1 / 2} \log ^{1 / 2} N+N^{\beta_{2}-1} J_{s}\right) .
$$




\section{A.1 Preliminaries}

Proof.According to Assumption (A5), it is trivial that

$$
\max _{1 \leq t \leq n} \max _{1 \leq j \leq N}\left|N^{-1} \sum_{i=1}^{j}\left(\varepsilon_{t i}-Z_{t i, \varepsilon}\right)\right|=\mathcal{O}_{\text {a.s. }}\left(N^{\beta_{2}-1}\right) .
$$

Next, the B spline basis satisfies

$$
\left|B_{\ell, p}\left(\frac{j}{N}\right)-B_{\ell, p}\left(\frac{j+1}{N}\right)\right| \leq N^{-1}\left\|B_{\ell, p}\right\|_{0,1} \leq C J_{s} N^{-1}
$$

uniformly over $1 \leq j \leq N$ and $1 \leq \ell \leq J_{s}+p$, while Assumptions (A2) and (A6) imply that $J_{s} N^{-1} \sim N^{\gamma} d_{N} N^{-1} \sim N^{\gamma-1} d_{N} \gg N^{-\nu}$, hence

$$
\left|\sigma\left(\frac{j}{N}\right)-\sigma\left(\frac{j+1}{N}\right)\right| \leq N^{-\nu}\|\sigma\|_{0, \nu} \leq C J_{s} N^{-1}
$$

uniformly over $1 \leq j \leq N$. Note that for $1 \leq \ell \leq J_{s}+p$, both $B_{\ell, p}(\cdot)$ and $\sigma(\cdot)$ are bounded on $[0,1]$, then

$$
\begin{aligned}
& \left|B_{\ell, p}\left(\frac{j}{N}\right) \sigma\left(\frac{j}{N}\right)-B_{\ell, p}\left(\frac{j+1}{N}\right) \sigma\left(\frac{j+1}{N}\right)\right| \\
& =\left|\left\{B_{\ell, p}\left(\frac{j}{N}\right)-B_{\ell, p}\left(\frac{j+1}{N}\right)+B_{\ell, p}\left(\frac{j+1}{N}\right)\right\} \sigma\left(\frac{j}{N}\right)-B_{\ell, p}\left(\frac{j+1}{N}\right) \sigma\left(\frac{j+1}{N}\right)\right| \\
& \leq\left|B_{\ell, p}\left(\frac{j}{N}\right)-B_{\ell, p}\left(\frac{j+1}{N}\right)\right| \sigma\left(\frac{j}{N}\right)+\left|\sigma\left(\frac{j}{N}\right)-\sigma\left(\frac{j+1}{N}\right)\right| B_{\ell, p}\left(\frac{j+1}{N}\right) \\
& \leq C J_{s} N^{-1} .
\end{aligned}
$$

Since the support of $B_{\ell, p}(\cdot)$ has length at most $p /\left(J_{s}+1\right)$, one obtains that

$$
\sum_{j=1}^{N-1}\left|B_{\ell, p}\left(\frac{j}{N}\right) \sigma\left(\frac{j}{N}\right)-B_{\ell, p}\left(\frac{j+1}{N}\right) \sigma\left(\frac{j+1}{N}\right)\right| \leq C
$$




\section{A.1 Preliminaries}

Hence,

$$
\begin{aligned}
\left|(n N)^{-1} \sum_{t=1}^{n} \sum_{j=1}^{N} B_{\ell, p}(j / N) \sigma(j / N)\left(\varepsilon_{t j}-Z_{t j, \varepsilon}\right)\right| \\
=\mid n^{-1} \sum_{t=1}^{n}\left[\sum_{j=1}^{N-1}\left\{B_{\ell, p}\left(\frac{j}{N}\right) \sigma\left(\frac{j}{N}\right)-B_{\ell, p}\left(\frac{j+1}{N}\right) \sigma\left(\frac{j+1}{N}\right)\right\} N^{-1} \sum_{i=1}^{j}\left(\varepsilon_{t i}-Z_{t i, \varepsilon}\right)\right] \\
+n^{-1} \sum_{t=1}^{n}\left\{B_{\ell, p}(1) \sigma(1) N^{-1} \sum_{j=1}^{N}\left(\varepsilon_{t j}-Z_{t j, \varepsilon}\right)\right\} \mid \\
\leq\left\{\max _{1 \leq t \leq n} \max _{1 \leq j \leq N}\left|N^{-1} \sum_{i=1}^{j}\left(\varepsilon_{t i}-Z_{t i, \varepsilon}\right)\right|\right\}\left\{\sum_{j=1}^{N-1}\left|B_{\ell, p}\left(\frac{j}{N}\right) \sigma\left(\frac{j}{N}\right)-B_{\ell, p}\left(\frac{j+1}{N}\right) \sigma\left(\frac{j+1}{N}\right)\right|\right\} \\
+C\left\{\max _{1 \leq t \leq n} \max _{1 \leq j \leq N}\left|N^{-1} \sum_{i=1}^{j}\left(\varepsilon_{t i}-Z_{t i, \varepsilon}\right)\right|\right\} \\
=\mathcal{O}_{a . s}\left\{N^{\beta_{2}-1}+N^{\beta_{2}-1}\right\}=\mathcal{O}_{a . s}\left(N^{\beta_{2}-1}\right) .
\end{aligned}
$$

Hence,

$$
\max _{1 \leq \ell \leq J_{s}+p}\left|(n N)^{-1} \sum_{t=1}^{n} \sum_{j=1}^{N} B_{\ell, p}(j / N) \sigma(j / N)\left(\varepsilon_{t j}-Z_{t j, \varepsilon}\right)\right|=\mathcal{O}_{a . s}\left(N^{\beta_{2}-1}\right) .
$$

The above inequality and Lemma A.6 together imply that

$\max _{1 \leq \ell \leq J_{s}+p}\left|(n N)^{-1} \sum_{t=1}^{n} \sum_{j=1}^{N} B_{\ell, p}(j / N) \sigma(j / N) \varepsilon_{t j}\right|=\mathcal{O}_{a . s .}\left(n^{-1 / 2} N^{-1 / 2} J_{s}^{-1 / 2} \log ^{1 / 2} N+N^{\beta_{2}-1}\right)$.

Clearly $(n N)^{-1} \mathbf{X}^{\top} \sum_{t=1}^{n} \varepsilon_{t}=\left\{(n N)^{-1} \sum_{t=1}^{n} \sum_{j=1}^{N} B_{\ell, p}(j / N) \sigma(j / N) \varepsilon_{t j}\right\}_{\ell=1}^{J_{s}+p}$,

thus

$$
\left\|(n N)^{-1} \mathbf{X}^{\top} \sum_{t=1}^{n} \varepsilon_{t}\right\|_{\infty}=\mathcal{O}_{a . s .}\left(n^{-1 / 2} N^{-1 / 2} J_{s}^{-1 / 2} \log ^{1 / 2} N+N^{\beta_{2}-1}\right) .
$$




\section{A.2 Proof of Theorem 2}

Recalling the definition of $\widetilde{\varepsilon}_{i}(x)$ in (3.21) and equation (3.17), the proof is completed by

$$
\begin{aligned}
\sup _{x \in[0,1]} n^{-1}\left|\sum_{t=1}^{n} \widetilde{\varepsilon}_{t}(x)\right| & =\left\|n^{-1} N^{-1} \mathbf{B}(x)^{\top} \mathbf{V}_{n, p}^{-1} \mathbf{X}^{\top} \sum_{t=1}^{n} \varepsilon_{t}\right\|_{\infty} \\
& =\mathcal{O}_{\text {a.s. }}\left(n^{-1 / 2} N^{-1 / 2} J_{s}^{1 / 2} \log ^{1 / 2} N+N^{\beta_{2}-1} J_{s}\right) .
\end{aligned}
$$

\section{A.2 Proof of Theorem 2}

For any $k=1,2, \ldots$, let $\phi_{k}=\left(\phi_{k}(1 / N), \ldots, \phi_{k}(N / N)\right)^{\top}$, and denote $\widetilde{\phi}_{k}(x)=N^{-1} \mathbf{B}(x)^{\top} \mathbf{V}_{n, p}^{-1} \mathbf{X}^{\top} \boldsymbol{\phi}_{k}$. According to (3.19), $\widetilde{\eta}_{t}(x)=\widetilde{m}(x)+\sum_{k=1}^{\infty} \xi_{t k} \widetilde{\phi}_{k}(x)$, therefore,

$$
\widetilde{\eta}_{t}(x)-\eta_{t}(x)=\widetilde{m}(x)-m(x)+\sum_{k=1}^{\infty} \xi_{t k}\left\{\widetilde{\phi}_{k}(x)-\phi_{k}(x)\right\}
$$

Lemma A.4 of Cao et al. (2012) provides a constant $C_{q, \mu}>0$, such that

$$
\begin{aligned}
& \|\widetilde{m}-m\|_{\infty} \leq C_{q, \mu}\|m\|_{q, \mu} J_{s}^{-p^{*}}, \\
& \left\|\widetilde{\phi}_{k}-\phi_{k}\right\|_{\infty} \leq C_{q, \mu}\left\|\phi_{k}\right\|_{q, \mu} J_{s}^{-p^{*}}, \quad k \geq 1
\end{aligned}
$$

Thus (A.5) and (A.6), and Assumption (A4) lead to

$$
\left\|\widetilde{\eta}_{t}-\eta_{t}\right\|_{\infty} \leq\|\widetilde{m}-m\|_{\infty}+\sum_{k=1}^{\infty}\left|\xi_{t k}\right|\left\|\widetilde{\phi}_{k}-\phi_{k}\right\|_{\infty} \leq C_{q, \mu} W_{t} J_{s}^{-p^{*}}
$$

where $W_{t}=\|m\|_{q, \mu}+\sum_{k=1}^{\infty}\left|\xi_{t k}\right|\left\|\phi_{k}\right\|_{q, \mu}, t=1, \ldots, n$, are identically distributed nonnegative random variables with finite mean. Assumption (A6) 


\section{A.2 Proof of Theorem 2}

then implies that

$$
\mathbb{P}\left\{\max _{1 \leq t \leq n} W_{t}>(n \log n)^{2 / r_{1}}\right\} \leq n \frac{\mathrm{E} W_{t}^{r_{1}}}{(n \log n)^{2}}=\mathrm{E} W_{t}^{r_{1}} n^{-1}(\log n)^{-2}
$$

thus,

$$
\sum_{n=1}^{\infty} \mathbb{P}\left\{\max _{1 \leq t \leq n} W_{t}>n \log n\right\} \leq \mathrm{E} W_{t}^{r_{1}} \sum_{n=1}^{\infty} n^{-1}(\log n)^{-2}<+\infty
$$

so $\max _{1 \leq t \leq n} W_{t}=\mathcal{O}_{a . s .}\left\{(n \log n)^{2 / r_{1}}\right\}, \max _{1 \leq t \leq n}\left\|\widetilde{\eta}_{t}-\eta_{t}\right\|_{\infty}=\mathcal{O}_{a . s .}\left\{J_{s}^{-p^{*}}(n \log n)^{2 / r_{1}}\right\}$,

thus,

$$
\left\|n^{-1} \sum_{t=1}^{n}\left\{\widetilde{\eta}_{t}(x)-\eta_{t}(x)\right\}\right\|_{\infty}=\mathcal{O}_{a . s .}\left\{J_{s}^{-p^{*}}(n \log n)^{2 / r_{1}}\right\} .
$$

By noticing that $\left\|n^{-1} \sum_{t=1}^{n} \widetilde{\varepsilon}_{t}(x)\right\|_{\infty}=\mathcal{O}_{a . s .}\left(n^{-1 / 2} N^{-1 / 2} J_{s}^{1 / 2} \log ^{1 / 2} N+N^{\beta_{2}-1} J_{s}\right)$

in Lemma A.7 and 3.22, one obtaions that

$$
\sup _{x \in[0,1]}|\bar{m}(x)-\widehat{m}(x)|=\mathcal{O}_{a . s .}\left\{J_{s}^{-p^{*}}(n \log n)^{2 / r_{1}}+n^{-1 / 2} N^{-1 / 2} J_{s}^{1 / 2} \log ^{1 / 2} N+N^{\beta_{2}-1} J_{s}\right\}
$$

The orders of $J_{s}$ and $n$ relative to $N$ in Assumptions (A3) and (A6) imply

that

$$
\sup _{x \in[0,1]} n^{1 / 2}|\bar{m}(x)-\widehat{m}(x)|=\mathcal{O}_{p}(1) .
$$

The proof is completed. 


\section{A.3 Proof of Theorem 1}

\section{A.3 Proof of Theorem 1}

Noting that $\operatorname{Cov}\left(Z_{j k, \xi}, Z_{j+h, k, \xi}\right)=\sum_{m=0}^{\infty} a_{m k} a_{m+h, k}, 1 \leq j \leq n, 1 \leq h \leq$ $n-j$ in Lemma A.5, it is easy to compute that

$$
\operatorname{Var}\left(\bar{Z}_{\cdot k, \xi}\right)=n^{-1}+2 n^{-2}\left\{\sum_{m=1}^{n-1} \sum_{t=0}^{\infty}(n-m) a_{t k} a_{t+m, k}\right\}
$$

Denote $\widetilde{\varphi}_{k}(x)=\bar{Z}_{\cdot k, \xi} \phi_{k}(x), k=1, \ldots, \infty$ and define $\varphi_{n}(x)=n^{1 / 2} G_{\varphi}(x, x)^{-1 / 2} \sum_{k=1}^{\infty} \widetilde{\varphi}_{k}(x)$, then for any $\left(x_{1}, \cdots, x_{l}\right) \in[0,1]^{l}$ and $\left(b_{1}, \cdots, b_{l}\right) \in \mathbb{R}^{l}$,

$$
\begin{aligned}
& \lim _{n \rightarrow \infty} \operatorname{Var}\left(\sum_{i=1}^{l} b_{i} \varphi_{n}\left(x_{i}\right)\right)=\lim _{n \rightarrow \infty} \operatorname{Var}\left(n^{1 / 2} \sum_{i=1}^{l} b_{i} G_{\varphi}\left(x_{i}, x_{i}\right)^{-1 / 2} \sum_{k=1}^{\infty} \bar{Z}_{\cdot k, \xi} \phi_{k}\left(x_{i}\right)\right) \\
= & \sum_{i=1}^{l} b_{i}^{2}+2 \sum_{1 \leq i<j \leq l} b_{i} b_{j} G_{\varphi}\left(x_{i}, x_{i}\right)^{-1 / 2} G_{\varphi}\left(x_{j}, x_{j}\right)^{-1 / 2} G_{\varphi}\left(x_{i}, x_{j}\right)=\operatorname{Var}\left(\sum_{i=1}^{l} b_{i} \varphi\left(x_{i}\right)\right) .
\end{aligned}
$$

Hence

$$
\left\{\varphi_{n}\left(x_{1}\right), \cdots, \varphi_{n}\left(x_{l}\right)\right\} \rightarrow_{D}\left\{\varphi\left(x_{1}\right), \cdots, \varphi\left(x_{l}\right)\right\}
$$

Assumption (A4) states that $G_{\varphi}(x, x) \geq C_{\varphi}>0, x \in[0,1]$, so $\omega\left(\varphi_{n}, \delta\right)$ satisfies

$$
\begin{aligned}
\omega\left(\varphi_{n}, \delta\right) & =\sup _{x, x^{\prime} \in[0,1],\left|x-x^{\prime}\right| \leq \delta}\left|\varphi_{n}(x)-\varphi_{n}\left(x^{\prime}\right)\right| \\
& \leq \sup _{x, x^{\prime} \in[0,1],\left|x-x^{\prime}\right| \leq \delta} n^{1 / 2} C_{\varphi}^{-1 / 2} \sum_{k=1}^{\infty}\left|\phi_{k}(x)-\phi_{k}\left(x^{\prime}\right)\right|\left|\bar{Z}_{\cdot k, \xi}\right| \\
& \leq n^{1 / 2} C_{\varphi}^{-1 / 2} \delta^{\mu} \sum_{k=1}^{\infty}\left\|\phi_{k}\right\|_{0, \mu}\left|\bar{Z}_{\cdot k, \xi}\right| .
\end{aligned}
$$




\section{A.3 Proof of Theorem 1}

Since $\mathbb{E}\left|\bar{Z}_{\cdot k, \xi}\right|=(2 / \pi)^{1 / 2} \operatorname{Var}\left(\bar{Z}_{\cdot k, \xi}\right)^{1 / 2}$, thus

$$
\begin{aligned}
\mathbb{P}\left[\omega\left(\varphi_{n}, \delta\right) \geq \epsilon\right] & \leq \mathbb{P}\left(n^{1 / 2} \delta^{\mu} C_{\varphi}^{-1 / 2} \sum_{k=1}^{\infty}\left\|\phi_{k}\right\|_{0, \mu}\left|\bar{Z}_{\cdot k, \xi}\right| \geq \epsilon\right) \\
& \leq \frac{(2 / \pi)^{1 / 2} \delta^{\mu} C_{\varphi}^{-1 / 2} \sum_{k=1}^{\infty}\left\|\phi_{k}\right\|_{0, \mu}\left\{n \operatorname{Var}\left(\bar{Z}_{\cdot k, \xi}\right)\right\}^{1 / 2}}{\epsilon}
\end{aligned}
$$

Since $\sum_{k=1}^{\infty}\left\|\phi_{k}\right\|_{0, \mu}<+\infty$ in Assumption (A4) and $n \operatorname{Var}\left(\bar{Z}_{\cdot k, \xi}\right) \rightarrow 1+$ $2 \sum_{t=0}^{\infty} \sum_{t^{\prime}=t+1}^{\infty} a_{t k} a_{t^{\prime} k}$ as $n \rightarrow \infty$, it is clear that

$$
\lim _{\delta \rightarrow 0} \limsup _{n \rightarrow \infty} \frac{(2 / \pi)^{1 / 2} \delta^{\mu} C_{\varphi}^{-1 / 2} \sum_{k=1}^{\infty}\left\|\phi_{k}\right\|_{0, \mu}\left\{n \operatorname{Var}\left(\bar{Z}_{\cdot k, \xi}\right)\right\}^{1 / 2}}{\epsilon}=0 .
$$

Thus

$$
\lim _{\delta \rightarrow 0} \limsup _{n \rightarrow \infty} \mathbb{P}\left[\omega\left(\varphi_{n}, \delta\right) \geq \epsilon\right]=0
$$

According to (A.7) and Lemma A.2, $\varphi_{n} \rightarrow_{D} \varphi$.

Note that

$$
\begin{aligned}
& n^{1 / 2} \sup _{x \in[0,1]} G_{\varphi}(x, x)^{-1 / 2}\left|\sum_{k=1}^{\infty}\left(\bar{Z}_{\cdot k, \xi}-\bar{\xi}_{\cdot k}\right) \phi_{k}(x)\right| \\
& \leq n^{1 / 2} \sup _{x \in[0,1]} G_{\varphi}(x, x)^{-1 / 2} \sum_{k=1}^{k_{n}}\left|\bar{Z}_{\cdot k, \xi}-\bar{\xi}_{\cdot k}\right|\left|\phi_{k}(x)\right| \\
& \quad+n^{1 / 2} \sup _{x \in[0,1]} G_{\varphi}(x, x)^{-1 / 2} \sum_{k=k_{n}+1}^{\infty}\left|\bar{Z}_{\cdot k, \xi}-\bar{\xi}_{\cdot k}\right|\left|\phi_{k}(x)\right| .
\end{aligned}
$$

Applying (A.3) leads to that

$$
\mathbb{P}\left\{\max _{1 \leq k \leq k_{n}}\left|\bar{\xi}_{\cdot k}-\bar{Z}_{\cdot k, \xi}\right|>C_{3} n^{\beta_{3}-1}\right\}<C_{4} n^{-\gamma_{3}}
$$




\section{A.3 Proof of Theorem 1}

By Borel Cantelli lemma, one has

$$
\max _{1 \leq k \leq k_{n}}\left|\bar{\xi}_{\cdot k}-\bar{Z}_{\cdot k, \xi}\right|=\mathcal{O}_{a . s .}\left(n^{\beta_{3}-1}\right)
$$

By Assumption (A4), $\sum_{k=1}^{\infty}\left\|\phi_{k}\right\|_{\infty}<+\infty$, thus $\sum_{k=1}^{k_{n}}\left\|\phi_{k}\right\|_{\infty}<C$ for some constant $C$. Together with (A.8) and Assumption (A3), one obtains that

$$
\begin{aligned}
& n^{1 / 2} \sup _{x \in[0,1]} G_{\varphi}(x, x)^{-1 / 2} \sum_{k=1}^{k_{n}}\left|\bar{Z}_{\cdot k, \xi}-\bar{\xi}_{\cdot k}\right|\left|\phi_{k}(x)\right| \\
& \leq n^{1 / 2} C_{G}^{-1 / 2} \sup _{x \in[0,1]} \sum_{k=1}^{k_{n}}\left|\phi_{k}(x)\right| \max _{1 \leq k \leq k_{n}}\left|\bar{\xi}_{\cdot k}-\bar{Z}_{\cdot k, \xi}\right| \\
& \leq n^{1 / 2} C_{G}^{-1 / 2} \sum_{k=1}^{k_{n}}\left\|\phi_{k}\right\|_{\infty} \max _{1 \leq k \leq k_{n}}\left|\bar{\xi}_{\cdot k}-\bar{Z}_{\cdot k, \xi}\right| \\
& \leq n^{1 / 2} C_{G}^{-1 / 2} C \mathcal{O}_{\text {a.s. }}\left(n^{\beta_{3}-1}\right)=\mathcal{O}_{\text {a.s. }}\left(n^{\beta_{3}-1 / 2}\right)=\mathcal{O}_{\text {a.s. }} \text { (1) }
\end{aligned}
$$

Note that

$\left(\mathbb{E}\left|\bar{\xi}_{\cdot k}\right|\right)^{2}=\left(\mathbb{E}\left|\bar{Z}_{\cdot k, \xi}\right|\right)^{2} \leq \mathbb{E} \bar{Z}_{\cdot k, \xi}^{2}=n^{-1}+2 n^{-2}\left\{\sum_{m=1}^{n-1} \sum_{t=0}^{\infty}(n-m) a_{t k} a_{t+m, k}\right\}$

thus $\mathbb{E}\left|\bar{\xi}_{\cdot k}\right|=\mathbb{E}\left|\bar{Z}_{\cdot k, \xi}\right|=\mathcal{O}\left(n^{-1 / 2}\right)$. In addition, Assumption (A4) states that $\sum_{k=k_{n}+1}^{\infty}\left\|\phi_{k}\right\|_{\infty}=\mathcal{O}\left(n^{-1 / 2}\right)$, then there exists

$$
\begin{aligned}
& \mathbb{E} n^{1 / 2} \sup _{x \in[0,1]} G_{\varphi}(x, x)^{-1 / 2} \sum_{k=k_{n}+1}^{\infty}\left|\bar{Z}_{\cdot k, \xi}-\bar{\xi}_{\cdot k}\right|\left|\phi_{k}(x)\right| \\
& \leq n^{1 / 2} C_{G}^{-1 / 2} \sum_{k=k_{n}+1}^{\infty}\left\|\phi_{k}\right\|_{\infty} \mathbb{E}\left|\bar{Z}_{\cdot k, \xi}-\bar{\xi}_{\cdot k}\right| \\
& \leq n^{1 / 2} C_{G}^{-1 / 2} \mathcal{O}\left(n^{-1 / 2}\right) \mathcal{O}\left(n^{-1 / 2}\right)=\mathcal{O}(1)
\end{aligned}
$$




\section{REFERENCES}

Combining (A.9) and (A.10), one has

$$
\mathbb{E} n^{1 / 2} \sup _{x \in[0,1]} G_{\varphi}(x, x)^{-1 / 2}\left|\sum_{k=1}^{\infty}\left(\bar{Z}_{\cdot k, \xi}-\bar{\xi}_{\cdot k}\right) \phi_{k}(x)\right|=\mathcal{O}(1),
$$

hence

$$
n^{1 / 2} \sup _{x \in[0,1]} G_{\varphi}(x, x)^{-1 / 2}\left|\sum_{k=1}^{\infty}\left(\bar{Z}_{\cdot k, \xi}-\bar{\xi}_{\cdot k}\right) \phi_{k}(x)\right|=\mathcal{O}_{p}(1) .
$$

Note that

$\varphi_{n}(x)-n^{1 / 2} G_{\varphi}(x, x)^{-1 / 2}\{\bar{m}(x)-m(x)\}=n^{1 / 2} G_{\varphi}(x, x)^{-1 / 2} \sum_{k=1}^{\infty}\left(\bar{Z}_{\cdot k, \xi}-\bar{\xi}_{\cdot k}\right) \phi_{k}(x)$,

hence

$$
\sup _{x \in[0,1]}\left|\varphi_{n}(x)-n^{1 / 2} G_{\varphi}(x, x)^{-1 / 2}\{\bar{m}(x)-m(x)\}\right|=\mathcal{O}_{p}(1) .
$$

The proof is completed by applying Slutsky's theorem.

\section{Acknowledgements}

This research is supported by National Natural Science Foundation of China awards 11771240 and 12026242 .

\section{References}

Billingsley, P. (1999). Convergence of Probability Measures. Wiley, New York.

Bosq, D. (2000). Linear Processes in Function Spaces: Theory and Applications. Springer- 
Brockwell, P.J. and Davis, R.A. (1991). Time Series: Theory and Methods, 2nd Edition. Springer, New York.

Cao, G., Wang, L., Li, Y. and Yang, L. (2016). Oracle-efficient confidence envelopes for covariance functions in dense functional data. Stat Sin 26, 359-383.

Cao, G., Yang, L. and Todem, D. (2012). Simultaneous inference for the mean function based on dense functional data. J Nonparametr Stat 24, 359-377.

Chen, M. and Song, Q. (2015). Simultaneous inference of the mean of functional time series. Electron. J. Stat. 9, 1779-1798.

Choi, H. and Reimherr, M. (2018). A geometric approach to confidence regions and bands for functional parameters. J. R. Stat. Soc. Ser. B Stat. Methodol. 80, 239-260.

Csőrgő, M., and Révész, P. (1981). Strong Approximations in Probability and Statistics. Academic Press, New York-London.

de Boor, C. (2001). A Practical Guide to Splines. Springer-Verlag, New York.

Degras, D.A. (2011). Simultaneous confidence bands for nonparametric regression with functional data. Stat Sin 21, 1735-1765.

DeVore, R. and Lorentz, G. (1993). Constructive Approximation: Polynomials and Splines Approximation. Springer-Verlag, Berlin.

Ferraty, F. and Vieu, P. (2006) Nonparametric Functional Data Analysis: Theory and Practice. Springer, New York. 


\section{REFERENCES}

Gu, L., Wang, L., Härdle, W. and Yang, L. (2014). A simultaneous confidence corridor for varying coefficient regression with sparse functional data. TEST 23, 806-843.

Gu, L. and Yang, L. (2015). Oracally efficient estimation for single-index link function with simultaneous confidence band. Electron. J. Stat. 9, 1540-1561.

Horváth, L., Kokoszka, P. and Reeder, R. (2013). Estimation of the mean of functional time series and a two-sample problem. J. R. Stat. Soc. Ser. B Stat. Methodol. 75, 103-122.

Hsing, T. and Eubank, R. (2015). Theoretical Foundations of Functional Data Analysis, with an Introduction to Linear Operators. Wiley, Chichester.

Kokoszka, P. and Reimherr, M. (2017). Introduction to Functional Data Analysis. Chapman \& Hall/CRC, Boca Raton.

Ma, S., Yang, L. and Carroll, R. (2012). A simultaneous confidence band for sparse longitudinal regression. Stat Sin 22, 95-122.

Ramsay, J. and Silverman, B. (2002). Applied Functional Data Analysis: Methods and Case Studies., Springer, New York.

Ramsay, J. and Silverman, B. (2005). Functional Data Analysis, Springer, New York.

Wang J. (2012). Modelling time trend via spline confidence band. Ann. Inst. Statist. Math. 64, $275-301$.

Wang, Y., Wang, G., Wang, L. and Ogden, T. (2020). Simultaneous confidence corridors for mean functions in functional data analysis of imaging data. Biometrics 76, 427-437. 


\section{REFERENCES}

Wang, J. and Yang, L. (2009). Polynomial spline confidence bands for regression curves. Stat $\operatorname{Sin}$ 19, 325-342.

Yu, S., Wang, L., Wang, G., Liu, C. and Yang, L. (2020). Estimation and inference for generalized geoadditive models. J Am Stat Assoc 115, 761-774.

Zheng, S., Liu, R., Yang, L. and Härdle, W. (2016). Statistical inference for generalized additive models: simultaneous confidence corridors and variable selection. TEST 25, 607-626.

Zheng, S., Yang, L. and Härdle, W. (2014). A smooth simultaneous confidence corridor for the mean of sparse functional data. J Am Stat Assoc 109, 661-673.

Center for Statistical Science and Department of Industrial Engineering, Tsinghua University, Beijing 100084, China.

E-mail: li-j17@mails.tsinghua.edu.cn

Center for Statistical Science and Department of Industrial Engineering, Tsinghua University, Beijing 100084, China.

E-mail: yanglijian@tsinghua.edu.cn 


\section{REFERENCES}

Table 1: Coverage frequencies of SCB in (4.25) with $p=4$, Case 1.

\begin{tabular}{|c|c|c|c|c|c|c|c|c|}
\hline \multirow[b]{2}{*}{$(n, N)$} & \multicolumn{4}{|c|}{$\sigma=0.3$} & \multicolumn{4}{|c|}{$\sigma=0.5$} \\
\hline & $\alpha=0.01$ & $\alpha=0.05$ & $\alpha=0.10$ & $\alpha=0.20$ & $\alpha=0.01$ & $\alpha=0.05$ & $\alpha=0.10$ & $\alpha=0.20$ \\
\hline$(100,120)$ & 0.962 & 0.897 & 0.840 & 0.708 & 0.957 & 0.896 & 0.831 & 0.692 \\
\hline$(400,500)$ & 0.974 & 0.921 & 0.871 & 0.770 & 0.971 & 0.919 & 0.867 & 0.763 \\
\hline$(900,1000)$ & 0.976 & 0.925 & 0.882 & 0.764 & 0.976 & 0.925 & 0.879 & 0.763 \\
\hline$(1600,2000)$ & 0.990 & 0.943 & 0.893 & 0.804 & 0.990 & 0.942 & 0.892 & 0.802 \\
\hline
\end{tabular}

Table 2: Coverage frequencies of SCB in (4.25) with $p=4$, Case 1.

\begin{tabular}{|ccccccccccc|}
\hline & \multicolumn{2}{c}{$\sigma(x)=(\exp (x)-0.9) /(\exp (x)+0.9)$} & & \multicolumn{3}{c|}{$\sigma(x)=0.1 \sin (2 \pi x)+0.2$} \\
$(n, N)$ & $\alpha=0.01$ & $\alpha=0.05$ & $\alpha=0.10$ & $\alpha=0.20$ & $\alpha=0.01$ & $\alpha=0.05$ & $\alpha=0.10$ & $\alpha=0.20$ \\
\hline$(100,120)$ & 0.960 & 0.895 & 0.837 & 0.702 & & 0.963 & 0.896 & 0.841 & 0.713 \\
$(400,500)$ & 0.959 & 0.911 & 0.845 & 0.748 & 0.961 & 0.912 & 0.847 & 0.748 \\
$(900,1000)$ & 0.984 & 0.930 & 0.882 & 0.752 & 0.984 & 0.912 & 0.862 & 0.740 \\
$(1600,2000)$ & 0.986 & 0.942 & 0.896 & 0.792 & 0.986 & 0.940 & 0.882 & 0.784 \\
\hline
\end{tabular}

Table 3: Coverage frequencies of SCB in (4.25) with $p=4$, Case 2.

\begin{tabular}{|ccccc|}
\hline$(n, N)$ & $\alpha=0.01$ & $\alpha=0.05$ & $\alpha=0.10$ & $\alpha=0.20$ \\
\hline$(100,500)$ & 0.966 & 0.882 & 0.826 & 0.722 \\
$(200,500)$ & 0.972 & 0.912 & 0.844 & 0.714 \\
$(300,500)$ & 0.976 & 0.920 & 0.854 & 0.766 \\
$(400,500)$ & 0.980 & 0.938 & 0.872 & 0.761 \\
\hline
\end{tabular}




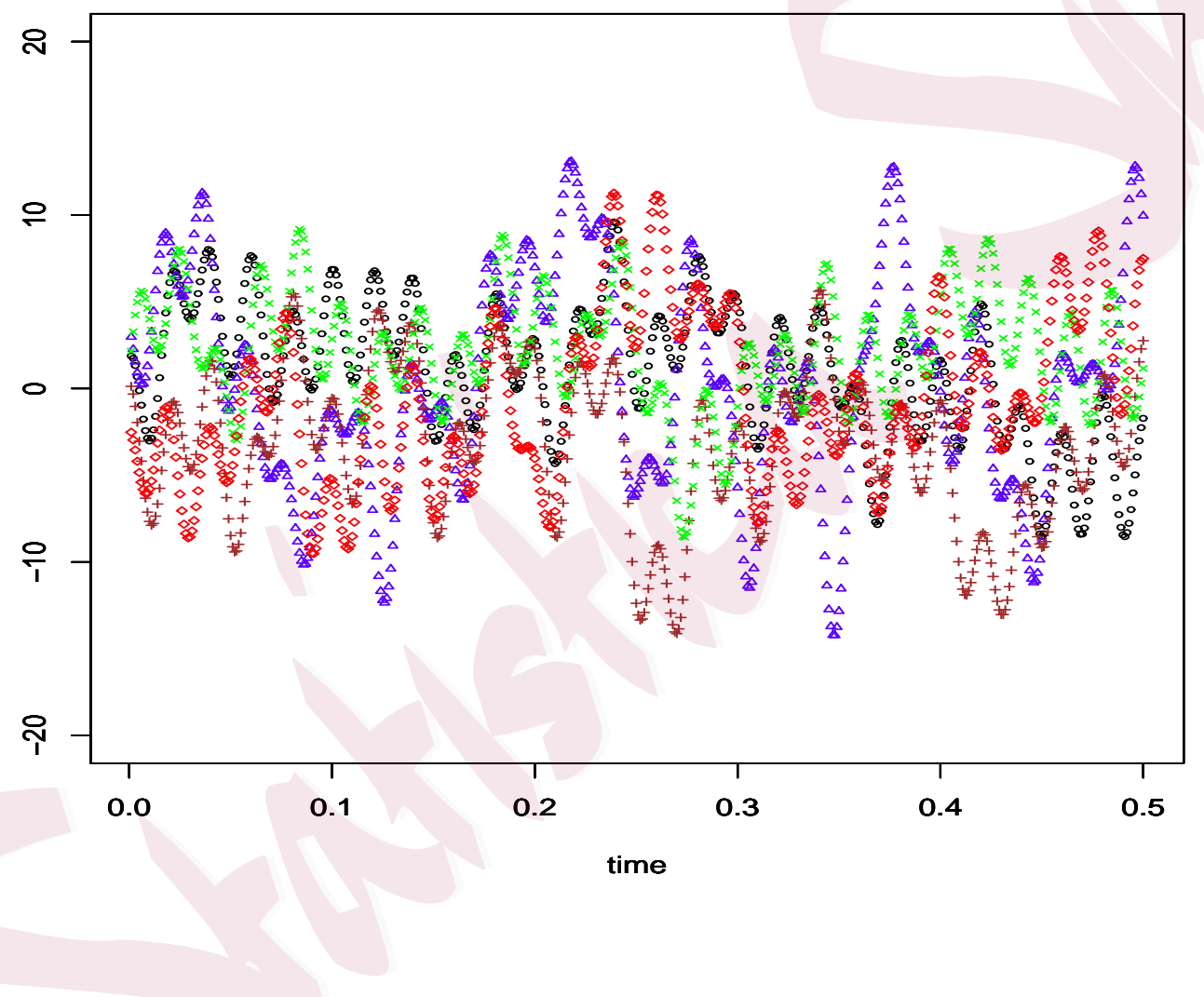

Figure 1: Five smoothed trajectories for the EEG data. 


\section{REFERENCES}

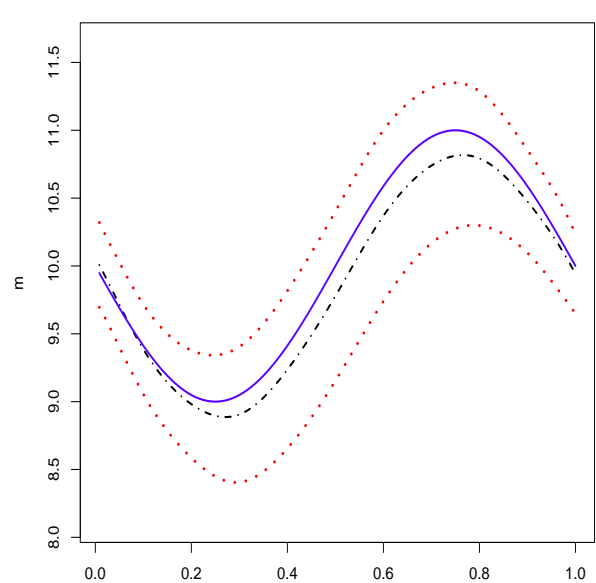

(a)

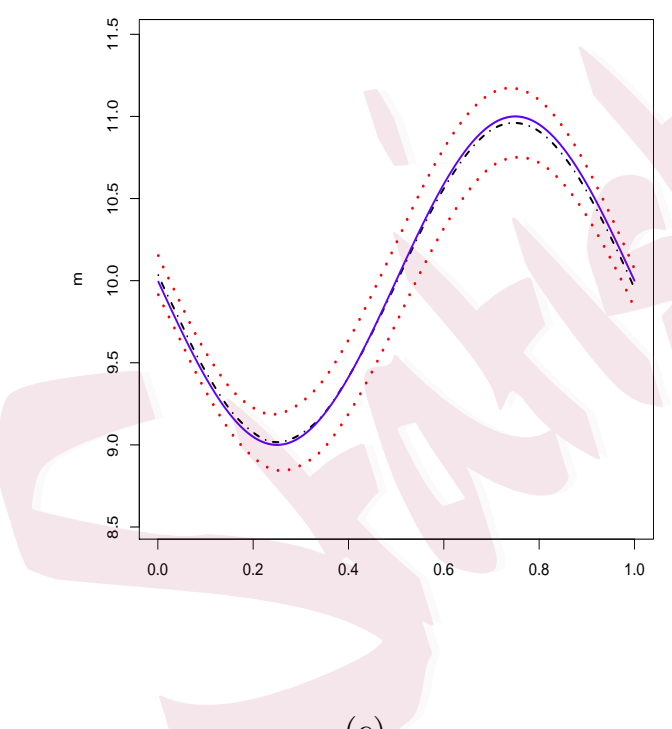

(c)

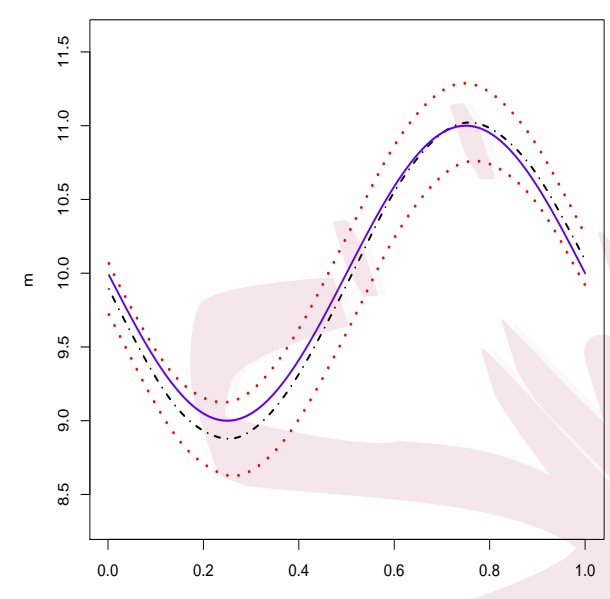

(b)

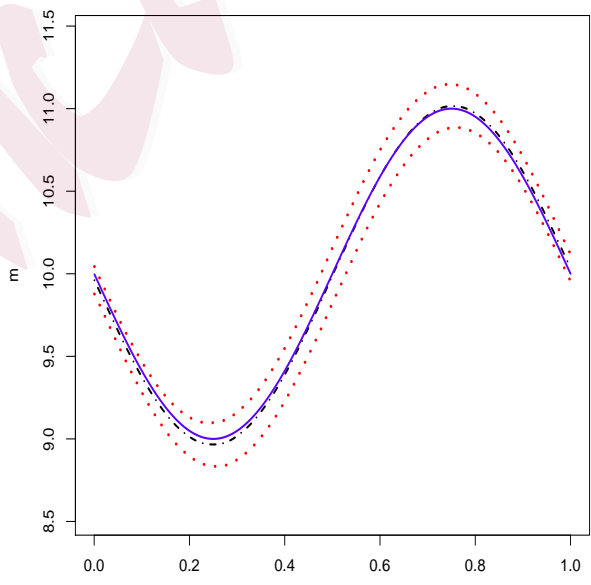

(d)

Figure 2: Plots of the cubic estimator in (2.9) for simulated data (dash) and 95\% SCBs in (4.25) (dotted), for $m(x)$ (solid). The number of observations $N$ of (a)-(d) are 100, 400, 900, 1600, respectively. In all panels, $\sigma=0.3$. 


\section{REFERENCES}

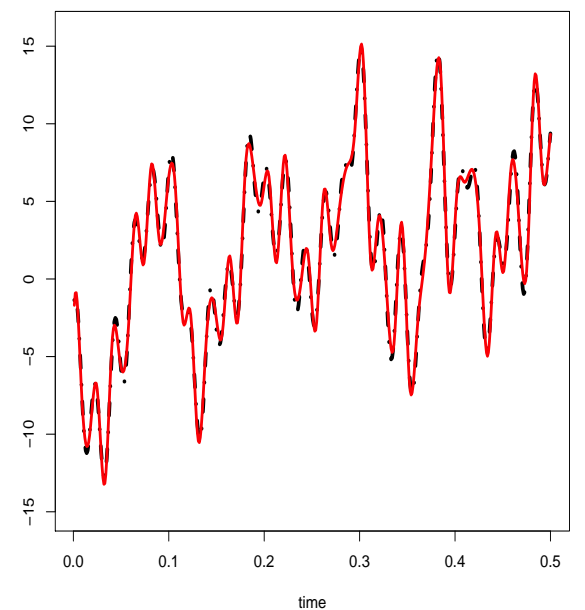

(a)

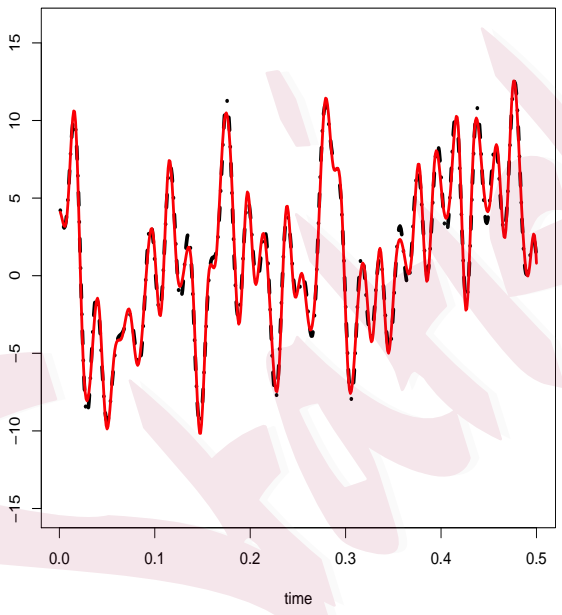

(c)

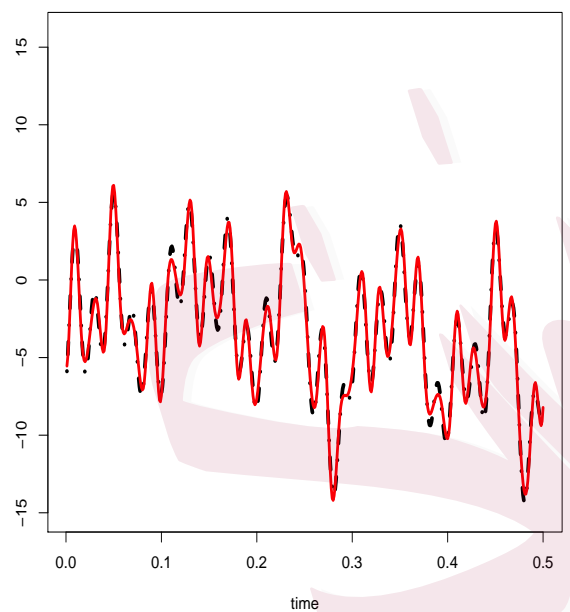

(b)

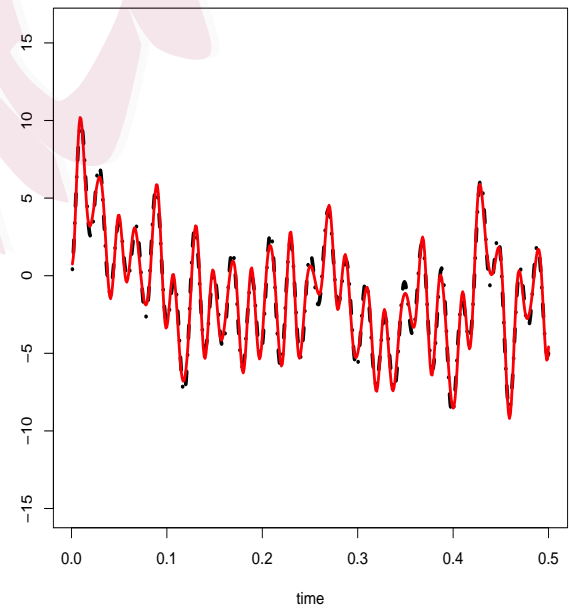

(d)

Figure 3: Plots of 4 randomly selected B spline trajectories (solid) together with their raw EEG data (dash). 


\section{REFERENCES}

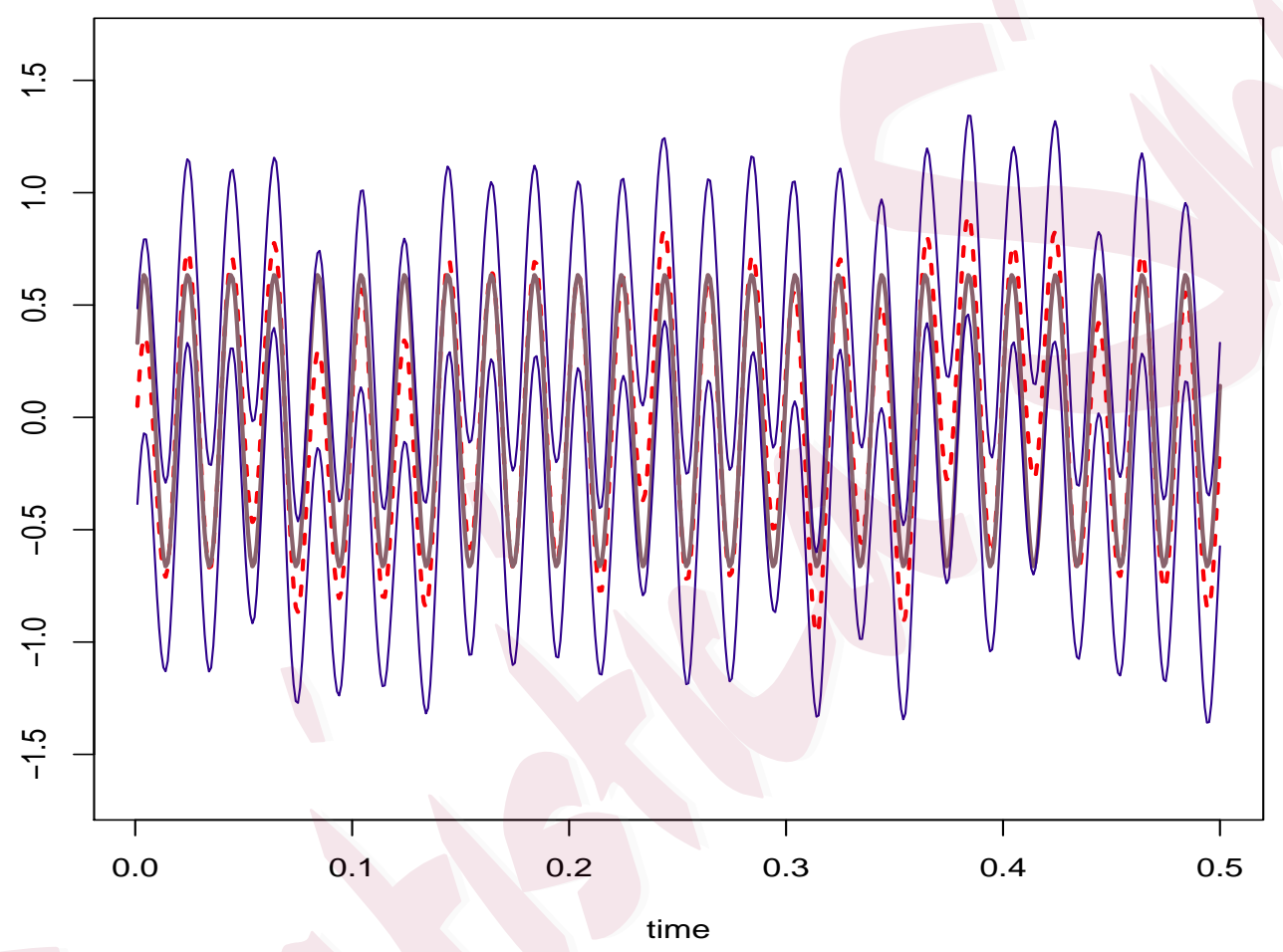

Figure 4: Plots of the null hypothesis curve $m_{0}(x)=-0.0148+$ $0.632 \sin (100 \pi x)+0.157 \cos (100 \pi x) \quad$ (thick), spline estimator $\widehat{m}(x)$ (dashed), $100(1-\alpha) \%=100(1-0.972) \%$ SCB (solid) for $m(x)$. 